\title{
Efficient Calculation of the Fields of a Dipole Radiating Above an Impedance Surface
}

\author{
Kamal Sarabandi, Fellow, IEEE, Mark D. Casciato, Member, IEEE, and Il-Suek Koh, Associate Member, IEEE
}

\begin{abstract}
In this paper, the classic problem of field computation for an infinitesimal dipole radiating above an impedance half-space is revisited. The expressions for the traditional solution consist of integrals of the Sommerfeld type that cannot be evaluated in closed form and due to their highly oscillatory nature are difficult to evaluate numerically. A method known as exact image theory, which has previously been applied to vertical electric and magnetic dipoles, is used to derive explicit expressions for dipoles of arbitrary orientation above impedance surfaces. Starting from the spectral representation of the field, the reflection coefficients are cast in the form of exact Laplace transforms and then by changing the order of integrations field expressions in terms of rapidly converging integrals are obtained. These expressions are exact, and valid for any arbitrary source alignment or observation position. It is shown that the formulation for a horizontal dipole contains an image in the conjugate complex plane resulting in a diverging exponential term not previously addressed in the literature. It is shown through further mathematical manipulations, that the diverging term is a contribution of the mirror image which can be extracted. Comparison of numerical results from exact image theory and the original Sommerfeld-type expressions shows good agreement as well as a speedup in computation time of many orders of magnitude, which depends on the distance between the transmitter and the receiver. This formulation can effectively replace the approximate asymptotic expressions used for predicting wave propagation over a smooth planar ground (having different regions of validity). The exact image formulation is also of practical use in evaluation of the Green's function for various applications in scattering problems where approximate solutions are not sufficient.
\end{abstract}

Index Terms-Dipole antennas, impedance boundary conditions.

\section{INTRODUCTION}

$\mathbf{T}$ HE ABILITY to accurately and efficiently predict the effects of natural features on radio-wave propagation is essential in the development and design of a communications system. Accurate simulation of the propagation environment allows for optimal system design in terms of parameters such as power consumption and cost minimization by determining the most efficient system configuration. The natural environment consists of various scattering and diffraction mechanisms that must be accounted for, including obstacles such as hills and mountains, forested areas, and the effects of a lossy earth.

Manuscript received March 10, 2000; revised August 1, 2001. This work was supported in part by the U.S. Army Research Office under Contract DAAH04-96-1-0377 and by the National Science Foundation under Contract ECS-99779376.

The authors are with the Radiation Laboratory, Department of Electrical Engineering and Computer Science, University of Michigan, Ann Arbor, MI 48109-2122 USA.

Digital Object Identifier 10.1109/TAP.2002.802160
The effects on the propagating wave by a lossy earth can be modeled locally as a flat impedance surface. These effects can be decomposed into the effects of the homogeneous surface and the effect caused by some impedance transition in the surface such as a river, sea/land interface, or swamp/dry land transition. The effect of the impedance transition was addressed by Sarabandi and Casciato [1] in an analytic fashion for a transition with a general one-dimensional (1-D) impedance variation and small dipole excitation. The effect of the homogeneous surface, which is the classic Sommerfeld problem of an infinitesimal electric dipole radiating above a lossy half-space, is the focus of this work.

The problem of a infinitesimal electric dipole radiating above a lossy half-space was originally formulated by Arnold Sommerfeld, in his classic work published in 1909 [2]. Since then, it is an understatement to say that this problem has received a significant amount of attention in the literature with literally hundreds of papers published on the subject. The inclusion of a sign error in the original work prompted much debate over several decades on the existence of a Zenneck type surface wave and its significance in the fields generated by a vertical electric dipole. The complete history of the problem is beyond the scope of this paper, but suffice to say that independent derivations by Weyl [3], Sommerfeld [4], Van der Pol and Niessen [5], and Wise [6] confirmed the sign error, although Sommerfeld himself never admitted to any error in the original 1909 work. The corrected formulation confirmed the existence of a surface wave for certain values of impedance and observation angles, but showed its contribution to the total field only significant within a certain range of distances, dependent on the impedance of the half-space (Sommerfeld numerical distance). Readers are referred to the work of Norton [7], [8] for a concise formulation of the problem, with the correct sign, and Baños [9] for a complete perspective of the historical development of the mathematics of the problem.

The Sommerfeld solution is expressed in terms of integrals of the Sommerfeld type, which cannot be evaluated in closed form and due to their highly oscillatory nature are difficult to evaluate numerically. Numerous techniques, both analytic and numeric, have been applied to evaluate the Sommerfeld integrals in an approximate fashion. To evaluate the Sommerfeld integrals analytically standard asymptotic techniques, such as the method of steepest descent (saddle-point method), are typically applied [10]-[12]. These techniques are valid when distance between source and observation is large and contributions from poles (surface wave) and branch cuts (lateral wave) must be accounted for when deforming the contour. For source and observation near the surface the direct and reflected waves (geometrical 
optics (GO) term, first-order saddle point) tend to cancel and higher-order terms in the asymptotic expansion are dominate. Note that these higher-order terms are typically referred to in the literature as a Norton Surface Wave [7], [8]. For a highly-lossy surface (small normalized impedance) the pole approaches the saddle point and their contributions cannot be separated. In this case, standard saddle-point techniques cannot be applied and an alternate asymptotic technique is necessary [10], [11]. To evaluate the Sommerfeld-type integrals numerically, in an approximate fashion, several techniques have been proposed. Parhami et al. [13] proposed a method, valid for a vertical electric dipole, in which the integration contour is deformed to the steepest descent path. The integral is then solved asymptotically when distance between image and observation is large, and numerically when this distance is small. Again poles and branch cuts must be accounted for when the contour deformation encounters them and the technique requires evaluation of Hankel functions of complex argument. Michalski [14] improved this method by proposing a variation in the way a branch cut is handled. Johnson and Dudley [15] proposed a method, valid for small distances between image and observation, in which an analytic technique is applied to reduce the oscillatory nature of the Sommerfeld integrand. While these techniques improve the convergence properties of the Sommerfeld-type integrals, they require transformations which increase the complexity of the formulation and, as in the case of the asymptotic solutions, are not valid for all source and observation positions, and electrical parameters.

As none of these analytical-numerical techniques are valid for general source orientation and observation location or arbitrary impedance values, a solution is sought which transforms the Sommerfeld-type expressions into a form that retains the rigor and generality of the original formulation, while improving the computational efficiency to a degree which makes evaluation of the resulting exact expressions practical from a numerical standpoint.

In order to improve the convergence properties of the Sommerfeld-type integrals, exact image theory is applied. In this method, an integral transform in the form of a Laplace transform, is applied to the reflection coefficients in the Sommerfeld-type integrals. This results in expressions consisting of a double integral, one in the original-spectral domain, and one in the Laplace domain. Application of appropriate identities allows for analytical evaluation of the integral in the spectral domain and the remaining integral expressions in the Laplace domain are dominated by a rapidly decaying exponential. These integral expressions are exact with no approximations made and the decaying exponential in the integrand results in significantly improved convergence properties over the original formulation. The form of these integral expressions can be interpreted as a distributed line source, located at the image point of the dipole source, and extending into the complex plane. Representation of the reflection coefficients in the Sommerfeld formulation in terms of a Laplace transform for a vertical electric dipole, was apparently first introduced by Van der Pol [16], and can also be seen in the work of Norton or Furutsu [8] and [17]. Their intent in applying this type of integral transformation was to simplify the asymptotic evaluation of the Sommerfeld formulation by modifying the integrand into a more well-behaved form.

The distributed image interpretation of a vertical dipole source above an impedance surface can be seen in the work of Arnold Sommerfeld [18]. Evaluating the Sommerfeld expressions as a type of distributed image is also seen in the work of Booker and Clemmow [19], while the Sommerfeld formulation is also seen in the work of Felsen and Marcuvitz [10], where the case of a vertical-electric dipole, represented as a distributed image source, and located in the complex plane is addressed. Keller, independently, derived at a similar solution [20]. Lindell and Alanen extended the technique to that of electric and magnetic dipoles of arbitrary orientation radiating above a dielectric half-space [21]-[23]. For the dielectric case, there is no exact transform for the reflection coefficient and the formulation by Lindell and Alanen for the vertical electric dipole involves a decomposition of the kernel function of the Laplace transform. For the case of a horizontal electric dipole, only a formal solution is presented, with no explicit expressions or detailed interpretation of image behavior. This might have been due to the fact that for a general half-space dielectric medium, exact analytical expressions for the image currents do not exist. However, such expressions can be obtained for impedance surfaces and the behavior of the image currents for arbitrary dipole orientation can be studied.

Lindell did examine the special case [24] of the impedance surface and the convergence properties of a general exponential integrand. He equated a diverging exponential term to a surface wave contribution, resulting from a pole in the reflection coefficients. He only examined in detail the case of a vertical electric dipole and noted a surface wave can only exist for an inductive surface, for this case.

In this paper, exact image theory is studied in detail for the problem of an electric dipole, of arbitrary orientation, above an impedance half-space. In Section II, a spectraldomain representation, which consists of Sommerfeld-type integrals, is given for the dipole-electric fields, along with appropriate identities necessary for the derivations that follow. Next, the exact image transformations are applied to the case of an infinitesimal electric current dipole radiating above an impedance half-space. As mentioned, integral expressions for the horizontal dipole show a diverging exponential term, which is not apparent in the formulation by Lindell and Alanen for a dielectric half-space [23]. In Section III, the exact image expressions are further simplified and it is shown that the diverging-exponential term vanishes. Section IV gives results, including a comparison of field quantities generated by evaluation of the exact image integrals, and the original Sommerfeld-type expressions. In addition, a timing comparison shows numerical evaluation of the exact image formulation to be several orders of magnitude faster than numerical evaluation of the corresponding Sommerfeld-type integrals. This makes the exact image formulation of practical use in integral equation (IE) techniques which require efficient numerical evaluation of the Green's function in various radiation and scattering problems. Section V summarizes this work and conclusions are drawn. 


\section{EXACT IMAGE FORMULATION}

Consider the problem geometry shown in Fig. 1. A small dipole carrying current $I$ with orientation $\hat{l}$ is radiating in free space above an infinite, homogeneous impedance plane. The characteristic impedance of free space and of the impedance plane are defined as $Z_{0}$ and $Z_{1}$, respectively. The total fields above the impedance plane propagate with propagation constant $k$ and can be decomposed into a direct wave and diffracted wave given by

$$
\mathbf{E}^{T}\left(\mathbf{r}, \mathbf{r}^{\prime}\right)=\mathbf{E}^{i}\left(\mathbf{r}, \mathbf{r}^{\prime}\right)+\mathbf{E}^{d}\left(\mathbf{r}, \mathbf{r}^{\prime}\right)
$$

where $|\mathbf{r}|=\sqrt{x^{2}+y^{2}+z^{2}}$ is the distance to the observation point and $\left|\mathbf{r}^{\prime}\right|=\sqrt{x^{\prime 2}+y^{\prime 2}+z^{\prime 2}}$ is the distance to the source location. Also in (1), superscripts $T, i$, and $d$ are indicative of the total, direct, and diffracted fields, respectively; the diffracted fields being the perturbation in the total fields caused by the impedance half-space. $\mathbf{E}^{i}\left(\mathbf{r}, \mathbf{r}^{\prime}\right)$ can be calculated directly and the expression for it is given in the Appendix. Of interest in this paper are the diffracted fields, expressions for which were originally derived by Sommerfeld [2]. As the focus of this work is the transformation of the these expressions into a form more compatible to numerical evaluation, the derivation will not be repeated here. Interested readers are referred to the Appendix for an explicit derivation of these expressions. The spectral representation of the diffracted electric fields of a dipole of orientation $\hat{l}$, where $\hat{l}=l_{x} \hat{x}+l_{y} \hat{y}+l_{z} \hat{z}$, located at the origin, and radiating above an infinite impedance plane are given by

$$
\begin{aligned}
\mathbf{E}^{d}\left(\mathbf{r}, \mathbf{r}^{\prime}\right) & k Z_{0} I \\
= & \hat{x} \int_{0}^{\infty} \frac{k_{\rho}}{2 k_{z}}\left\{\Gamma _ { h } \left[-l_{x}\left(J_{2}\left(k_{\rho} \rho\right) \cos 2 \phi\right.\right.\right. \\
& \left.\left.\left.+J_{0}\left(k_{\rho} \rho\right)\right)-l_{y} J_{2}\left(k_{\rho} \rho\right) \sin 2 \phi\right)\right] \\
& +\Gamma_{v}\left[\frac{2 i k_{z} k_{\rho}}{k^{2}} l_{z} \cos \phi J_{1}\left(k_{\rho} \rho\right)+\frac{k_{z}^{2}}{k^{2}} l_{x}\right. \\
& +\left(J_{0}\left(k_{\rho} \rho\right)-J_{2}\left(k_{\rho} \rho\right) \cos 2 \phi\right) \\
& \left.\left.-\frac{k_{z}^{2}}{k^{2}} l_{y} J_{2}\left(k_{\rho} \rho\right) \sin 2 \phi\right]\right\} e^{i k_{z}\left(z+z^{\prime}\right)} d k_{\rho} \\
& +\hat{y} \int_{0}^{\infty} \frac{k_{\rho}}{2 k_{z}}\left\{\Gamma _ { h } \left[-l_{x} J_{2}\left(k_{\rho} \rho\right) \sin 2 \phi\right.\right. \\
& \left.-l_{y}\left(J_{0}\left(k_{\rho} \rho\right)-J_{2}\left(k_{\rho} \rho\right) \cos 2 \phi\right)\right] \\
& +\Gamma_{v}\left[\frac{2 i k_{z} k_{\rho}}{k^{2}} l_{z} \sin \phi J_{1}\left(k_{\rho} \rho\right)-\frac{k_{z}^{2}}{k^{2}} l_{x} J_{2}\left(k_{\rho} \rho\right) \sin 2 \phi\right. \\
& \left.\left.+\frac{k_{z}^{2}}{k^{2}} l_{y}\left(J_{0}\left(k_{\rho} \rho\right)+J_{2}\left(k_{\rho} \rho\right) \cos 2 \phi\right)\right]\right\} e^{i k_{z}\left(z+z^{\prime}\right)} d k_{\rho} \\
& -\hat{z} \int_{0}^{\infty} \frac{k_{\rho}}{k_{z}} \Gamma_{v}\left[\frac{k_{\rho}^{2}}{k^{2}} l_{z} J_{0}\left(k_{\rho} \rho\right)+\frac{i k_{z} k_{\rho}}{k^{2}}\right. \\
& \left.\left.+\left(l_{x} \cos \phi+l_{y} \sin \phi\right) J_{1}\left(k_{\rho} \rho\right)\right] e^{i k_{z}\left(z+z^{\prime}\right)} d k_{\rho}\right\}
\end{aligned}
$$

where $\Gamma_{h}$ and $\Gamma_{v}$ are the horizontal (TE to $z$ ) and vertical (TM to $z$ ) Fresnel reflection coefficients, respectively given by

$$
\Gamma_{h}=\frac{\eta-k / k_{z}}{\eta+k / k_{z}}, \quad \Gamma_{v}=\frac{-\eta+k_{z} / k}{\eta+k_{z} / k} .
$$

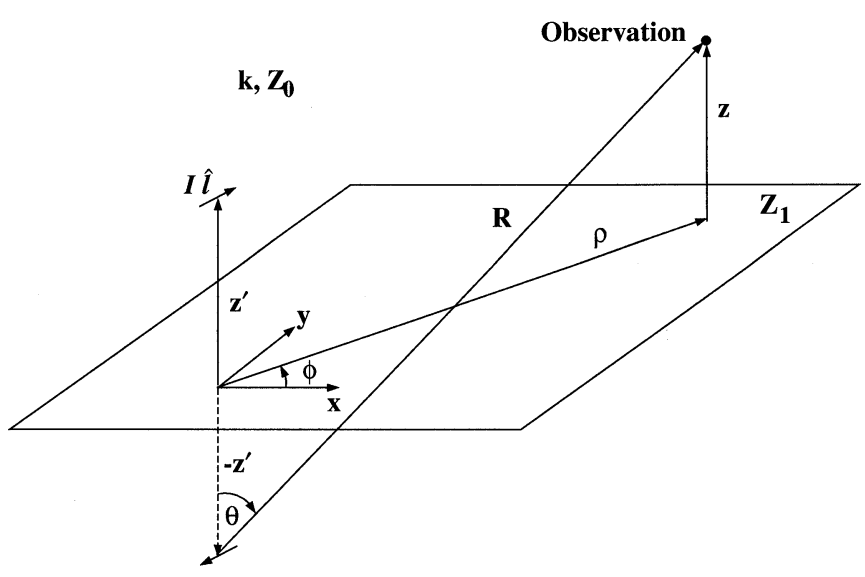

Fig. 1. Problem geometry, dipole above an impedance plane.

Here $\eta=Z_{1} / Z_{0}=\sqrt{1 /\left(\varepsilon^{\prime}+i \varepsilon^{\prime \prime}\right)}$ is the normalized impedance of the half-space and $\varepsilon_{r}=\varepsilon^{\prime}+i \varepsilon^{\prime \prime}$ is the relative complex permittivity of the lower half-space, approximated by an impedance surface. In (2), $k_{z}$ is the dependent variable defined as $k_{z}^{2}=k^{2}-k_{\rho}^{2}$, and $J_{0}, J_{1}$, and $J_{2}$ are Bessel functions of order 0,1 , and 2 , respectively. Also in (2), $\rho$ defines the radial distance between source and observation points, $z$ the height of the observation point, $\phi$ is the angle between $\rho$ and the $x$ axis, with $z^{\prime}$ being the height of the source point, all of which are seen in Fig. 1.

The integrals in (2) are Sommerfeld-type integrals and as already stated, they are highly oscillatory in nature with poor convergence properties making them difficult to evaluate numerically, especially for the case of $\rho \gg\left(z+z^{\prime}\right)$. To improve the convergence behavior of these integrals, exact image theory is applied by the application of integral transforms and appropriate identities. The method is exact with no approximations made and the resulting expressions are valid for any arbitrary source and observation position. The basic methodology is to first rewrite the spectral domain formulation of (2) in terms of zeroth-order Bessel functions only. Terms containing reflection coefficients in the resulting expressions are expanded where necessary, and then rewritten in the form of a simple Laplace transform. Order of integration is then exchanged and the spectral domain integration over $k_{\rho}$ in (2) is performed in an analytic fashion. The remaining expressions in the Laplace domain contain integrals which are dominated by rapidly decaying exponentials and exhibit significantly improved convergence properties over the original Sommerfeld expressions. In Section II-A, relevant transforms and identities will be given that are needed for the derivations that follow.

\section{A. Transforms and Identities}

In this section, transforms and identities that are used throughout the derivations that follow will be defined. To apply exact image theory, $\Gamma_{h}$ and $\Gamma_{v}$ given in (3) must be defined in terms of a Laplace transform. $\Gamma_{h}$ and $\Gamma_{v}$ can be rewritten as

$$
\Gamma_{h}=1-\frac{2 k / \eta}{k_{z}+k / \eta}=1-\frac{2 \alpha}{k_{z}+\alpha}
$$


where $\alpha=k / \eta$ and

$$
\Gamma_{v}=1-\frac{2 \eta k}{k_{z}+\eta k}=1-\frac{2 \beta}{k_{z}+\beta}
$$

where $\beta=\eta k$. Now recognizing that

$$
\int_{0}^{\infty} e^{-\gamma \xi} e^{-k_{z} \xi} d \xi=\frac{1}{k_{z}+\gamma}
$$

where $\gamma$ is some constant coefficient, we can rewrite $\Gamma_{h}$ and $\Gamma_{v}$ in the form of a Laplace transform or

$$
\Gamma_{h}=1-2 \alpha \int_{0}^{\infty} e^{-\left(\alpha+k_{z}\right) \xi} d \xi
$$

and

$$
\Gamma_{v}=1-2 \beta \int_{0}^{\infty} e^{-\left(\beta+k_{z}\right) \xi} d \xi
$$

In the application of exact image theory to the case of a horizontal dipole, several terms arise in the derivation which contain $\Gamma_{h}$ and $\Gamma_{v}$, however, they cannot be directly expressed as the Laplace transform defined in (6). For the sake of simplicity and in order to minimize the complexity of the resulting expressions, it is desirable that all integral transforms applied be in the form of this Laplace transform. For a horizontal dipole, this is accomplished by expanding the terms where necessary by partial fractions into a form that allows them to be directly written in the form of (6).

In applying exact image theory to the Sommerfeld-type expressions for the fields of a dipole above an impedance surface, an initial step in the derivation is to apply Bessel function identities in order to rewrite (2) in terms of $J_{0}$ only. To do this, the following identities are needed:

$$
\begin{aligned}
& \frac{1}{k^{2}} \frac{\partial^{2}}{\partial x^{2}} J_{0}\left(k_{\rho} \rho\right)=-\frac{k_{\rho}^{2}}{2 k^{2}}\left[J_{0}\left(k_{\rho} \rho\right)-\cos 2 \phi\right.\left.\cdot J_{2}\left(k_{\rho} \rho\right)\right] \\
& \frac{1}{k^{2}} \frac{\partial^{2}}{\partial y^{2}} J_{0}\left(k_{\rho} \rho\right)=-\frac{k_{\rho}^{2}}{2 k^{2}}\left[J_{0}\left(k_{\rho} \rho\right)+\cos 2 \phi\right.\left.\cdot J_{2}\left(k_{\rho} \rho\right)\right] \\
& \frac{1}{k^{2}}\left(\frac{\partial^{2}}{\partial x^{2}}+\frac{\partial^{2}}{\partial y^{2}}\right) J_{0}\left(k_{\rho} \rho\right)=-\frac{k_{\rho}^{2}}{k^{2}} J_{0}\left(k_{\rho} \rho\right) \\
& \frac{1}{k^{2}} \frac{\partial^{2}}{\partial x \partial y} J_{0}\left(k_{\rho} \rho\right)=\frac{k_{\rho}^{2}}{2 k^{2}} \sin 2 \phi J_{2}\left(k_{\rho} \rho\right) \\
& \frac{1}{k^{2}} \frac{\partial^{2}}{\partial x \partial z} J_{0}\left(k_{\rho} \rho\right)=-i \cos \phi \frac{k_{z} k_{\rho}}{k^{2}} J_{1}\left(k_{\rho} \rho\right) \\
& \frac{1}{k^{2}} \frac{\partial^{2}}{\partial y \partial z} J_{0}\left(k_{\rho} \rho\right)=-i \sin \phi \frac{k_{z} k_{\rho}}{k^{2}} J_{1}\left(k_{\rho} \rho\right) .
\end{aligned}
$$

An additional identity that will allow for the analytic evaluation of the spectral-domain integrals over $k_{\rho}$ in the exact image expressions is

$$
\frac{e^{i k R}}{R}=i \int_{0}^{\infty} J_{0}\left(k_{\rho} \rho\right) e^{i k_{z}\left(z+z^{\prime}\right)} \frac{k_{\rho}}{k_{z}} d k_{\rho}
$$

where $R=\sqrt{\left(x-x^{\prime}\right)^{2}+\left(y-y^{\prime}\right)^{2}+\left(z+z^{\prime}\right)^{2}}$. The identity in (15) relates the free-space Green's function to an alternate representation in the form of a Sommerfeld integral and is appropriately referred to in the literature as the Sommerfeldintegral identity.

\section{B. Vertical-Electric Dipole}

To find the diffracted fields generated by a vertical $(z$ directed) dipole, the components of (2) containing $l_{z}$ are first considered and are modified to include only zeroth-order Bessel functions. The reflection coefficients are rewritten in terms of their Laplace transform and then the order of integration is changed in order to evaluate the integral in terms of $k_{\rho}$ analytically, using the Sommerfeld-integral identity given by (15).

For a vertical dipole (2) reduces to

$$
\begin{aligned}
\mathbf{E}_{v}^{d}\left(\mathbf{r}, \mathbf{r}^{\prime}\right)= & \frac{k Z_{0} I}{4 \pi} l_{z} \int_{0}^{\infty} \frac{k_{\rho}}{k_{z}} \Gamma_{v}\left\{\frac{i k_{z} k_{\rho}}{k^{2}} \cos \phi J_{1}\left(k_{\rho} \rho\right) \hat{x}\right. \\
& \left.+\frac{i k_{z} k_{\rho}}{k^{2}} \sin \phi J_{1}\left(k_{\rho} \rho\right) \hat{y}-\frac{k_{\rho}^{2}}{k^{2}} J_{0}\left(k_{\rho} \rho\right) \hat{z}\right\} \\
& \cdot e^{i k_{z}\left(z+z^{\prime}\right)} d k_{\rho}
\end{aligned}
$$

where the subscript $v$ in (16) designates a vertical dipole. Applying the identities in Section II-A (16) can be rewritten as

$$
\begin{array}{r}
\mathbf{E}_{v}^{d}\left(\mathbf{r}, \mathbf{r}^{\prime}\right)=-\frac{Z_{0} I}{4 \pi k} l_{z}\left\{\frac{\partial^{2}}{\partial x \partial z} \hat{x}+\frac{\partial^{2}}{\partial y \partial z} \hat{y}-\left(\frac{\partial^{2}}{\partial x^{2}}+\frac{\partial^{2}}{\partial y^{2}}\right) \hat{z}\right\} \\
\cdot \int_{0}^{\infty} \frac{k_{\rho}}{k_{z}} \Gamma_{v} J_{0}\left(k_{\rho} \rho\right) e^{i k_{z}\left(z+z^{\prime}\right)} d k_{\rho} .
\end{array}
$$

Now rewriting $\Gamma_{v}$ in the form of (8) and substituting into (17) gives

$$
\begin{aligned}
\mathbf{E}_{v}^{d}\left(\mathbf{r}, \mathbf{r}^{\prime}\right) & \\
=- & \frac{Z_{0} I}{4 \pi k} l_{z}\left\{\frac{\partial^{2}}{\partial x \partial z} \hat{x}+\frac{\partial^{2}}{\partial y \partial z} \hat{y}-\left(\frac{\partial^{2}}{\partial x^{2}}+\frac{\partial^{2}}{\partial y^{2}}\right) \hat{z}\right\} \\
& \cdot\left[\int_{0}^{\infty} \frac{k_{\rho}}{k_{z}} J_{0}\left(k_{\rho} \rho\right) e^{i k_{z}(z+z)} d k_{\rho}-2 \beta \int_{0}^{\infty} \int_{0}^{\infty} \frac{k_{\rho}}{k_{z}} J_{0}\left(k_{\rho} \rho\right)\right. \\
& \left.\cdot e^{-\beta \xi} e^{i k_{z}\left(z+z^{\prime}+i \xi\right)} d k_{\rho} d \xi\right] .
\end{aligned}
$$

The integrals in (18) in terms of $k_{\rho}$ can be solved analytically by applying (15), giving the final form of the diffracted-electric fields for a vertical ( $z$ directed) dipole

$$
\begin{aligned}
& \mathbf{E}_{v}^{d}\left(\mathbf{r}, \mathbf{r}^{\prime}\right)=\frac{i Z_{0} I}{4 \pi k} l_{z}\left\{\frac{\partial^{2}}{\partial x \partial z} \hat{x}+\frac{\partial^{2}}{\partial y \partial z} \hat{y}-\left(\frac{\partial^{2}}{\partial x^{2}}+\frac{\partial^{2}}{\partial y^{2}}\right) \hat{z}\right\} \\
& \cdot\left[\frac{e^{i k R}}{R}-2 \beta \int_{0}^{\infty} e^{-\beta \xi} \frac{e^{i k R^{\prime}(\xi)}}{R^{\prime}(\xi)} d \xi\right]
\end{aligned}
$$

where $R$ is as previously defined and $R^{\prime}(\xi)=$ $\sqrt{\left(x-x^{\prime}\right)^{2}+\left(y-y^{\prime}\right)^{2}+\left(z+z^{\prime}+i \xi\right)^{2}}$. The integrand in the last term of (19) can be interpreted as a distributed image source in the complex $z$ plane, located at $-z^{\prime}$ as seen in Fig. 2. In this integrand, both exponential factors $-\beta \xi$ and $k R^{\prime}(\xi)$ decay rapidly as $\xi$ becomes large. Due to this, the integral in (19) converges very rapidly, for all source and observation locations. 


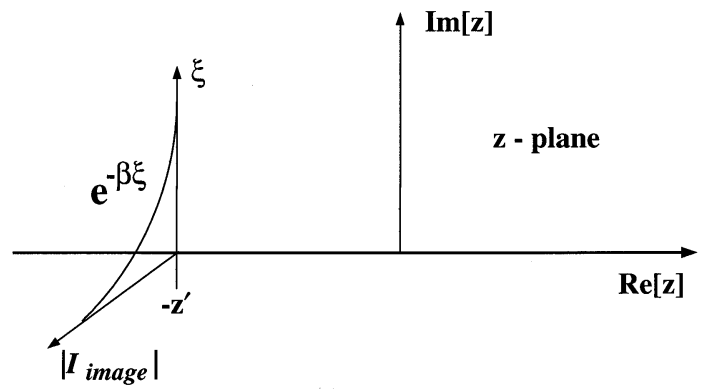

Fig. 2. Exact image in $z$ plane.

\section{Horizontal Electric Dipole}

In a manner similar to that of the vertical dipole, appropriate terms in the Sommerfeld-type expressions for a horizontal dipole will be put in terms of $J_{0}$ only to facilitate evaluation of the integral over $k_{\rho}$ analytically. Initially, the $x$ component of the electric field is derived. The $y$ component is determined in the same fashion, which for brevity will not be repeated and only the final result is provided. Finally, the $z$ component of the field generated by a horizontal-electric dipole is derived.

To derive the $x$ component of the diffracted-electric field for a horizontal electric dipole, the identities of (9)-(14) are applied, and noting that $k_{z}^{2}=k^{2}-k_{\rho}^{2}$

$$
\begin{aligned}
\mathbf{E}_{h}^{d}\left(\mathbf{r}, \mathbf{r}^{\prime}\right) \cdot \hat{x} & \\
= & \frac{k Z_{0} I}{4 \pi} \int_{0}^{\infty}\left[\frac{l_{x}}{k_{\rho}^{2}}\left(\Gamma_{h} \frac{\partial^{2}}{\partial y^{2}}-\Gamma_{v} \frac{k^{2}-k_{\rho}^{2}}{k^{2}} \frac{\partial^{2}}{\partial x^{2}}\right)\right. \\
& \left.-\frac{l_{y}}{k_{\rho}^{2}}\left(\Gamma_{h}+\Gamma_{v} \frac{k^{2}-k_{\rho}^{2}}{k^{2}}\right) \frac{\partial^{2}}{\partial x \partial y}\right] \frac{k_{\rho}}{k_{z}} J_{0}\left(k_{\rho} \rho\right) \\
& \cdot e^{i k_{z}\left(z+z^{\prime}\right)} d k_{\rho}
\end{aligned}
$$

where the subscript $h$ designates a horizontal dipole. Equation (20) can be rewritten as

$$
\begin{aligned}
\mathbf{E}_{h}^{d}\left(\mathbf{r}, \mathbf{r}^{\prime}\right) \cdot \hat{x}=\frac{k Z_{0} I}{4 \pi} & \left\{l_{x}\left(\frac{\partial^{2}}{\partial y^{2}} f_{a}-\frac{\partial^{2}}{\partial x^{2}} f_{b}+\frac{1}{k^{2}} \frac{\partial^{2}}{\partial x^{2}} f_{c}\right)\right. \\
& \left.-l_{y} \frac{\partial^{2}}{\partial x \partial y}\left(f_{a}+f_{b}-\frac{1}{k^{2}} f_{c}\right)\right\}
\end{aligned}
$$

where

and

$$
\begin{aligned}
f_{a} & =\int_{0}^{\infty} \frac{k_{\rho}}{k_{z}} J_{0}\left(k_{\rho} \rho\right) e^{i k_{z}\left(z+z^{\prime}\right)} \frac{\Gamma_{h}}{k_{\rho}^{2}} d k_{\rho} \\
f_{b} & =\int_{0}^{\infty} \frac{k_{\rho}}{k_{z}} J_{0}\left(k_{\rho} \rho\right) e^{i k_{z}\left(z+z^{\prime}\right)} \frac{\Gamma_{v}}{k_{\rho}^{2}} d k_{\rho}
\end{aligned}
$$

$$
f_{c}=\int_{0}^{\infty} \frac{k_{\rho}}{k_{z}} J_{0}\left(k_{\rho} \rho\right) e^{i k_{z}\left(z+z^{\prime}\right)} \Gamma_{v} d k_{\rho} .
$$

While $f_{c}$ can be evaluated directly by expressing $\Gamma_{v}$ in the form of (8) as in [(17)-(19), Sec. II-B], the terms $\Gamma_{h} / k_{\rho}^{2}$ and $\Gamma_{v} / k_{\rho}^{2}$ in $f_{a}$ and $f_{b}$, cannot be directly written in terms of the Laplace transform of (6), however, by applying partial fraction expan- sion they can be put in the appropriate form. Defining these terms as

$$
a=\frac{\Gamma_{h}}{k_{\rho}^{2}}, \quad b=\frac{\Gamma_{v}}{k_{\rho}^{2}}
$$

writing $\Gamma_{h}$ and $\Gamma_{v}$ explicitly in terms of $k, k_{z}, \alpha$, and $\beta$ [see (4) and (5)], and recognizing that $k_{\rho}^{2}=k^{2}-k_{z}^{2}=\left(k-k_{z}\right)\left(k+k_{z}\right)$, $a$ and $b$ can be written as

$$
a=\frac{k_{z}-\alpha}{\left(k-k_{z}\right)\left(k+k_{z}\right)\left(k_{z}+\alpha\right)}
$$

and

$$
b=\frac{k_{z}-\beta}{\left(k-k_{z}\right)\left(k+k_{z}\right)\left(k_{z}+\beta\right)} .
$$

Expanding (26) and (27) by partial fractions gives expressions of the following form for $a$ and $b$ :

$$
\begin{aligned}
& a=\frac{a_{1}}{\left(k-k_{z}\right)}+\frac{a_{2}}{\left(k+k_{z}\right)}+\frac{a_{3}}{\left(k_{z}+\alpha\right)} \\
& b=\frac{b_{1}}{\left(k-k_{z}\right)}+\frac{b_{2}}{\left(k+k_{z}\right)}+\frac{b_{3}}{\left(k_{z}+\beta\right)}
\end{aligned}
$$

where

$$
\begin{aligned}
& a_{1}=-b_{1}=\frac{\eta-1}{2 k(\eta+1)} \\
& a_{2}=-b_{2}=\frac{\eta+1}{2 k(\eta-1)} \\
& a_{3}=-b_{3}=\frac{2 \eta}{k\left(1-\eta^{2}\right)}
\end{aligned}
$$

where the coefficients in (30) are given explicitly in terms of normalized impedance $\eta$ and $k$, which will be used in the final expressions for the $x$ component of the diffracted electric fields. Now representing each term of the partial fraction expansions of (28) and (29), in the form of the Laplace transform of (6), changing the order of integration as before, and applying (15) to evaluate the integral over $k_{\rho}$ analytically, gives the following expressions for $f_{a}$ and $f_{b}$ :

$$
\begin{aligned}
& f_{a}=-i \int_{0}^{\infty}\left[e^{-k \xi}\left(a_{1} \frac{e^{i k R^{\prime \prime}(\xi)}}{R^{\prime \prime}(\xi)}+a_{2} \frac{e^{i k R^{\prime}(\xi)}}{R^{\prime}(\xi)}\right)\right. \\
& \left.+a_{3} e^{-\alpha \xi} \frac{e^{i k R^{\prime}(\xi)}}{R^{\prime}(\xi)}\right] d \xi \\
& f_{b}=-i \int_{0}^{\infty}\left[e^{-k \xi}\left(b_{1} \frac{e^{i k R^{\prime \prime}(\xi)}}{R^{\prime \prime}(\xi)}+b_{2} \frac{e^{i k R^{\prime}(\xi)}}{R^{\prime}(\xi)}\right)\right. \\
& \left.+b_{3} e^{-\beta \xi} \frac{e^{i k R^{\prime}(\xi)}}{R^{\prime}(\xi)}\right] d \xi .
\end{aligned}
$$

where $R^{\prime \prime}(\xi)=\sqrt{\left(x-x^{\prime}\right)^{2}+\left(y-y^{\prime}\right)^{2}+\left(z+z^{\prime}-i \xi\right)^{2}}$. Also, the expression for $f_{c}$ is given by

$$
f_{c}=-i\left\{\frac{e^{i k R}}{R}-2 \beta \int_{0}^{\infty} e^{-\beta \xi} \frac{e^{i k R^{\prime}(\xi)}}{R^{\prime}(\xi)} d \xi\right\} .
$$


Noting that $b=-a,(21)$ can be rewritten as

$$
\begin{aligned}
\mathbf{E}_{h}^{d}\left(\mathbf{r}, \mathbf{r}^{\prime}\right) \cdot \hat{x} & i k Z_{0} I\left\{l _ { x } ( \frac { \partial ^ { 2 } } { \partial x ^ { 2 } } + \frac { \partial ^ { 2 } } { \partial y ^ { 2 } } ) \int _ { 0 } ^ { \infty } \left[\frac{1-\eta}{2 k(1+\eta)} e^{-k \xi} \frac{e^{i k R^{\prime \prime}(\xi)}}{4 \pi R^{\prime \prime}(\xi)}\right.\right. \\
& \left.+\frac{1+\eta}{2 k(1-\eta)} e^{-k \xi} \frac{e^{i k R^{\prime}(\xi)}}{4 \pi R^{\prime}(\xi)}\right] d \xi-l_{x} \frac{2 \eta}{k\left(1-\eta^{2}\right)} \int_{0}^{\infty} \\
& \cdot\left[e^{-\alpha \xi} \frac{\partial^{2}}{\partial y^{2}} \frac{e^{i k R^{\prime}(\xi)}}{4 \pi R^{\prime}(\xi)}+e^{-\beta \xi} \frac{\partial^{2}}{\partial x^{2}} \frac{e^{i k R^{\prime}(\xi)}}{4 \pi R^{\prime}(\xi)}\right] d \xi \\
& -\frac{1}{k^{2}}\left(l_{x} \frac{\partial^{2}}{\partial x^{2}}+l_{y} \frac{\partial^{2}}{\partial x \partial y}\right)\left[\frac{e^{i k R}}{4 \pi R}-2 k \eta \int_{0}^{\infty}\right. \\
& \left.\cdot e^{-\beta \xi} \frac{e^{i k R^{\prime}(\xi)}}{4 \pi R^{\prime}(\xi)} d \xi\right]+l_{y} \frac{\partial^{2}}{\partial x \partial y}\left(\frac{2 \eta}{k\left(1-\eta^{2}\right)}\right) \int_{0}^{\infty} \\
& \left.\cdot\left(e^{-\alpha \xi}-e^{-\beta \xi}\right) \frac{e^{i k R^{\prime}(\xi)}}{4 \pi R^{\prime}(\xi)} d \xi\right\} \cdot
\end{aligned}
$$

In (34), an additional term is observed containing the exponential factor $R^{\prime \prime}(\xi)$, which is not present in the expressions for the vertical dipole and also is not evident in the formulation by Lindell and Alanen for a horizontal dipole over a dielectric half-space [23]. This denotes the image location in the conjugate complex $z$ plane that results in an exponentially diverging factor. While this term is an exponentially growing term, the image current distribution, which depends on the surface impedance, is exponentially decaying at a greater rate and dominates the integrand $\left(\xi>\operatorname{Im}\left[R^{\prime \prime}(\xi)\right]\right)$. Because of this, the integral in the first term of (34) still exhibits the rapid convergence properties inherent in the integral expressions generated by the application of exact image theory.

The $y$ component of the diffracted electric field generated by a horizontal dipole is derived in a similar fashion and for the sake of brevity is not repeated here. The expression for it is given by

$$
\begin{aligned}
\mathbf{E}_{h}^{d}\left(\mathbf{r}, \mathbf{r}^{\prime}\right) \cdot \hat{y} & \\
= & i k Z_{0} I\left\{l _ { y } ( \frac { \partial ^ { 2 } } { \partial x ^ { 2 } } + \frac { \partial ^ { 2 } } { \partial y ^ { 2 } } ) \int _ { 0 } ^ { \infty } \left[\frac{1-\eta}{2 k(1+\eta)} e^{-k \xi} \frac{e^{i k R^{\prime \prime}(\xi)}}{4 \pi R^{\prime \prime}(\xi)}\right.\right. \\
& \left.+\frac{1+\eta}{2 k(1-\eta)} e^{-k \xi} \frac{e^{i k R^{\prime}(\xi)}}{4 \pi R^{\prime}(\xi)}\right] d \xi-l_{y} \frac{2 \eta}{k\left(1-\eta^{2}\right)} \int_{0}^{\infty} \\
& \cdot\left[e^{-\alpha \xi} \frac{\partial^{2}}{\partial x^{2}} \frac{e^{i k R^{\prime}(\xi)}}{4 \pi R^{\prime}(\xi)}+e^{-\beta \xi} \frac{\partial^{2}}{\partial y^{2}} \frac{e^{i k R^{\prime}(\xi)}}{4 \pi R^{\prime}(\xi)}\right] d \xi \\
& -\frac{1}{k^{2}}\left(l_{y} \frac{\partial^{2}}{\partial y^{2}}+l_{x} \frac{\partial^{2}}{\partial x \partial y}\right)\left[\frac{e^{i k R}}{4 \pi R}-2 k \eta \int_{0}^{\infty}\right. \\
& \left.\cdot e^{-\beta \xi} \frac{e^{i k R^{\prime}(\xi)}}{4 \pi R^{\prime}(\xi)} d \xi\right]+l_{x} \frac{\partial^{2}}{\partial x \partial y}\left(\frac{2 \eta}{k\left(1-\eta^{2}\right)}\right) \int_{0}^{\infty} \\
& \left.\cdot\left(e^{-\alpha \xi}-e^{-\beta \xi}\right) \frac{e^{i k R^{\prime}(\xi)}}{4 \pi R^{\prime}(\xi)} d \xi\right\} \cdot
\end{aligned}
$$

Derivation of the $z$ component of the diffracted electric field generated by a horizontal dipole is rather straightforward. Starting from

$$
\begin{array}{r}
\mathbf{E}_{h}^{d}\left(\mathbf{r}, \mathbf{r}^{\prime}\right) \cdot \hat{z}=\frac{1}{k^{2}}\left(l_{x} \frac{\partial^{2}}{\partial x \partial z}+l_{y} \frac{\partial^{2}}{\partial y \partial z}\right) \int_{0}^{\infty} \frac{k_{\rho}}{k_{z}} \Gamma_{v} J_{0}\left(k_{\rho} \rho\right) \\
\cdot e^{i k_{z}\left(z+z^{\prime}\right)} d k_{\rho}
\end{array}
$$

and recognizing the integral in (36) as $f_{c}$, the $z$ component of the electric field generated by a horizontal dipole is given by

$$
\begin{aligned}
\mathbf{E}_{h}^{d}\left(\mathbf{r}, \mathbf{r}^{\prime}\right) \cdot \hat{z}= & -i k Z_{0} I\left\{\frac{1}{k^{2}}\left(l_{x} \frac{\partial^{2}}{\partial x \partial z}+l_{y} \frac{\partial^{2}}{\partial y \partial z}\right)\right. \\
& \left.\cdot\left[\frac{e^{i k R}}{4 \pi R}-2 \beta \int_{0}^{\infty} e^{-\beta \xi} \frac{e^{i k R^{\prime}(\xi)}}{4 \pi R^{\prime}(\xi)} d \xi\right]\right\} .
\end{aligned}
$$

\section{SimplificAtion OF THE EXACT-IMAGE Formulation}

To explain the peculiar behavior of the exponentially diverging image current for a horizontal dipole, an alternative representation is derived in this section. It is shown that the exponentially diverging image current represents the contribution from the mirror image (or perfect conductor), which can be extracted. The exact image formulation, derived in the previous sections, can be further simplified by observing the behavior of the $x$ component of the electric field expressions for the perfect electric conducting (PEC) case, $(\eta=0)$. For the PEC case (19), and (34) reduce to

$$
\begin{aligned}
\mathbf{E}^{d}\left(\mathbf{r}, \mathbf{r}^{\prime}\right) \cdot \hat{x}= & \frac{i Z_{0} I}{k}\left[-l_{x} \frac{\partial^{2}}{\partial x^{2}}-l_{y} \frac{\partial^{2}}{\partial x \partial y}+l_{z} \frac{\partial^{2}}{\partial x \partial z}\right] \frac{e^{i k R}}{4 \pi R} \\
& +\frac{i Z_{0} I}{2} l_{x}\left(\frac{\partial^{2}}{\partial x^{2}}+\frac{\partial^{2}}{\partial y^{2}}\right) \int_{0}^{\infty} \\
& \cdot e^{-k \xi}\left[\frac{e^{i k R^{\prime \prime}}}{4 \pi R^{\prime \prime}}+\frac{e^{i k R^{\prime}}}{4 \pi R^{\prime}}\right] d \xi
\end{aligned}
$$

According to image theory, this diffracted-field component should be equal to the $x$ component of the electric field radiated from an image dipole, given by

$$
\begin{aligned}
& \mathbf{E}^{i s}\left(\mathbf{r}, \mathbf{r}^{\prime}\right) \cdot \hat{x}=\frac{i Z_{0} I}{k} \\
& \cdot\left[-l_{x}\left(k^{2}+\frac{\partial^{2}}{\partial x^{2}}\right)-l_{y} \frac{\partial^{2}}{\partial x \partial y}+l_{z} \frac{\partial^{2}}{\partial x \partial z}\right] \frac{e^{i k R}}{4 \pi R}
\end{aligned}
$$

where the superscript is implies image source. Setting (38) equal to (39) the following relationship is obtained:

$$
\begin{aligned}
\left(\frac{\partial^{2}}{\partial x^{2}}\right. & \left.+\frac{\partial^{2}}{\partial y^{2}}\right) \int_{0}^{\infty} e^{-k \xi} \frac{e^{i k R^{\prime \prime}}}{4 \pi R^{\prime \prime}} d \xi \\
& =-2 k \frac{e^{i k R}}{4 \pi R}-\left(\frac{\partial^{2}}{\partial x^{2}}+\frac{\partial^{2}}{\partial y^{2}}\right) \int_{0}^{\infty} \frac{e^{i k R^{\prime}}}{4 \pi R^{\prime}} e^{-k \xi} d \xi
\end{aligned}
$$


Applying (40), and after some algebraic manipulation the expressions for diffracted fields [(19), (34), (35), and (37)] can be rewritten in a more compact form as

$$
\begin{gathered}
\mathbf{E}^{d}\left(\mathbf{r}, \mathbf{r}^{\prime}\right) \\
=\mathbf{E}^{i s}\left(\mathbf{r}, \mathbf{r}^{\prime}\right)+\mathbf{E}^{e s}\left(\mathbf{r}, \mathbf{r}^{\prime}\right)+\frac{2 i k Z_{0} I \eta}{1-\eta^{2}} \\
\cdot\left[l _ { x } \hat { x } \left\{\frac{\partial^{2}}{\partial x^{2}} \int_{0}^{\infty}\left(e^{-k \xi}-\eta^{2} e^{-\beta \xi}\right) \frac{e^{i k R^{\prime}}}{4 \pi R^{\prime}} d \xi\right.\right. \\
\left.+\frac{\partial^{2}}{\partial y^{2}} \int_{0}^{\infty}\left(e^{-k \xi}-e^{-\alpha \xi}\right) \frac{e^{i k R^{\prime}}}{4 \pi R^{\prime}} d \xi\right\} \\
+l_{y} \hat{y}\left\{\frac{\partial^{2}}{\partial x^{2}} \int_{0}^{\infty}\left(e^{-k \xi}-e^{-\alpha \xi}\right) \frac{e^{i k R^{\prime}}}{4 \pi R^{\prime}} d \xi\right. \\
\left.+\frac{\partial^{2}}{\partial y^{2}} \int_{0}^{\infty}\left(e^{-k \xi}-\eta^{2} e^{-\beta \xi}\right) \frac{e^{i k R^{\prime}}}{4 \pi R^{\prime}} d \xi\right\} \\
\left.+\frac{\partial^{2}}{\partial x \partial y} \int_{0}^{\infty}\left(e^{-\alpha \xi}-\eta^{2} e^{-\beta \xi}\right) \frac{e^{i k R^{\prime}}}{4 \pi R^{\prime}} d \xi\left(l_{y} \hat{x}+l_{x} \hat{y}\right)\right] \\
+2 i Z_{0} I \eta l_{z}\left(\frac{\partial^{2}}{\partial x \partial z} \hat{x}+\frac{\partial^{2}}{\partial y \partial z} \hat{y}\right) \int_{0}^{\infty} e^{-\beta \xi} \frac{e^{i k R^{\prime}}}{4 \pi R^{\prime}} d \xi \\
+2 i Z_{0} \eta \hat{z}\left[l_{x} \frac{\partial^{2}}{\partial x \partial z}+l_{y} \frac{\partial^{2}}{\partial y \partial z}+l_{z}\left(\frac{\partial^{2}}{\partial x^{2}}+\frac{\partial^{2}}{\partial y^{2}}\right)\right] \int_{0}^{\infty} \\
+e^{-\beta \xi} \frac{e^{i k R^{\prime}}}{4 \pi R^{\prime}} d \xi
\end{gathered}
$$

where $\mathbf{E}^{i s}\left(\mathbf{r}, \mathbf{r}^{\prime}\right)$ is the electric field radiated from the image source and given in (39), and $\mathbf{E}^{e s}\left(\mathbf{r}, \mathbf{r}^{\prime}\right)$, is given by

$$
\frac{2 i k Z_{0} I \eta}{1+\eta} \frac{e^{i k R}}{4 \pi R}\left(l_{x} \hat{x}+l_{y} \hat{y}\right)
$$

and can be interpreted as the electric field radiated from an electrostatic image source.

To further simplify the diffracted-field expressions in (41), consider the integral

$$
I=\int_{0}^{\infty} e^{-p \xi} \frac{e^{i k R^{\prime}}}{4 \pi R^{\prime}} d \xi .
$$

Integrating $I$ by parts and using the identity

$$
\frac{\partial}{\partial \xi} \frac{e^{i k R^{\prime}}}{4 \pi R^{\prime}}=i \frac{\partial}{\partial z} \frac{e^{i k R^{\prime}}}{4 \pi R^{\prime}}
$$

an analytic expression in terms of the derivative of $I$ with respect to $z$ is obtained

$$
I=-\frac{1}{p} \int_{0}^{\infty} \frac{e^{i k R^{\prime}}}{4 \pi R^{\prime}} \frac{\partial}{\partial \xi} e^{-p \xi} d \xi=\frac{1}{p}\left(\frac{e^{i k R}}{4 \pi R}+i \frac{\partial I}{\partial z}\right) .
$$

Therefore

$$
\frac{\partial I}{\partial z}=i \frac{e^{i k R}}{4 \pi R}-i p I .
$$

Taking the derivative of (42) with respect to $z$ and using (43), it can easily be shown that

$$
I=\left(i \frac{\partial}{\partial z}+p\right) \frac{e^{i k R}}{4 \pi R}-p^{2} I .
$$

In a source-free region, according to the Helmholtz equation

$$
\left(\frac{\partial^{2}}{\partial x^{2}}+\frac{\partial^{2}}{\partial y^{2}}+\frac{\partial^{2}}{\partial z^{2}}+k^{2}\right) \frac{e^{i k R^{\prime}}}{4 \pi R^{\prime}}=0
$$

This expression can be used to simplify (41) by replacing expressions containing $\left(\partial^{2} / \partial x^{2}\right)+\left(\partial^{2} / \partial y^{2}\right)$ with $-\left(\partial^{2} / \partial z^{2}\right)-$ $k^{2}$. Applying the identities of (43) and (44), the first two lines in (41) are reduced to

$$
-\left(\frac{\partial^{2}}{\partial z^{2}}+k^{2}\right) f_{1}\left(l_{x} \hat{x}+l_{y} \hat{y}\right)+\nabla_{t} \nabla_{t} \cdot\left(f_{2} \hat{l}\right)
$$

where

$$
\begin{aligned}
& \nabla_{t}=\frac{\partial}{\partial x} \hat{x}+\frac{\partial}{\partial y} \hat{y}, \quad f_{1}=\int_{0}^{\infty}\left(e^{-k \xi}-e^{-\alpha \xi}\right) \frac{e^{i k R^{\prime}}}{4 \pi R^{\prime}} d \xi, \\
& \text { and } \\
& \qquad f_{2}=\int_{0}^{\infty}\left(e^{-\alpha \xi}-\eta^{2} e^{-\beta \xi}\right) \frac{e^{i k R^{\prime}}}{4 \pi R^{\prime}} d \xi .
\end{aligned}
$$

In order to evaluate the $\partial^{2} / \partial x \partial z$ and $\partial^{2} / \partial y \partial z$ terms in (41), they are rewritten as

$$
\begin{aligned}
& \left(\frac{\partial^{2}}{\partial x \partial z} \hat{x}+\frac{\partial^{2}}{\partial y \partial z} \hat{y}\right) \int_{0}^{\infty} e^{-\beta \xi} \frac{e^{i k R^{\prime}}}{4 \pi R^{\prime}} d \xi \\
& \quad=\nabla_{t} \frac{\partial}{\partial z} \int_{0}^{\infty} e^{-\beta \xi} \frac{e^{i k R^{\prime}}}{4 \pi R^{\prime}} d \xi \\
& \quad=i \nabla_{t}\left(\frac{e^{i k R}}{4 \pi R}-\beta \int_{0}^{\infty} e^{-\beta \xi} \frac{e^{i k R^{\prime}}}{4 \pi R^{\prime}} d \xi\right) .
\end{aligned}
$$

Applying (43) and (44) to (45) and (46), the diffracted field expressions of (41), can be expressed as

$$
\begin{aligned}
\mathbf{E}^{d}\left(\mathbf{r}, \mathbf{r}^{\prime}\right) & \\
= & \mathbf{E}^{i s}\left(\mathbf{r}, \mathbf{r}^{\prime}\right)+\mathbf{E}^{e s}\left(\mathbf{r}, \mathbf{r}^{\prime}\right)-\frac{2 i Z_{0} I \eta}{1-\eta^{2}}\left(l_{x} \hat{x}+l_{y} \hat{y}\right) \\
& \cdot\left[(k-\alpha) \frac{e^{i k R}}{4 \pi R}-\left(k^{2}-\alpha^{2}\right) \int_{0}^{\infty} e^{-\alpha \xi} \frac{e^{i k R^{\prime}}}{4 \pi R^{\prime}} d \xi\right] \\
& +2 Z_{0} I \eta l_{z} \nabla \frac{e^{i k R}}{4 \pi R}-2 i Z_{0} I \eta l_{z} \hat{z} \\
& \cdot\left[\beta \frac{e^{i k r}}{4 \pi r}+\left(k^{2}-\beta^{2}\right) \int_{0}^{\infty} e^{-\beta \xi} \frac{e^{i k R^{\prime}}}{4 \pi R^{\prime}} d \xi\right] \\
& +\frac{2 i Z_{0} I \eta}{1-\eta^{2}} \nabla_{t} \nabla_{t} \cdot\left(f_{2} \hat{l}\right)-2 Z_{0} \eta \\
& \cdot\left[\hat{z} \nabla_{t}\left(\frac{e^{i k R}}{4 \pi R} \hat{l}\right)+\beta\left\{l_{z} \nabla_{t} f_{3}-\hat{z} \nabla_{t} \cdot\left(f_{3} \hat{l}\right)\right\}\right]
\end{aligned}
$$

where

$$
f_{3}=\int_{0}^{\infty} e^{-\beta \xi} \frac{e^{i k R^{\prime}}}{4 \pi R^{\prime}} d \xi
$$

Now by applying the identity $\hat{l} \times(a \hat{x}-b \hat{y})=b\left(l_{z} \hat{x}-l_{x} \hat{z}\right)+$ $a\left(l_{z} \hat{y}-l_{y} \hat{z}\right)$, the last term in (47) can be rewritten as

$$
l_{z} \nabla_{t} f_{3}-\hat{z} \nabla_{t} \cdot\left(f_{3} \hat{l}\right)=\hat{l} \times\left(\frac{\partial}{\partial y} \hat{x}-\frac{\partial}{\partial x} \hat{y}\right) f_{3} .
$$


The final simplified form of the exact image expressions for the diffracted field from a small dipole of arbitrary orientation are given by

$$
\begin{aligned}
\mathbf{E}^{d}\left(\mathbf{r}, \mathbf{r}^{\prime}\right) & \\
= & \mathbf{E}^{i s}\left(\mathbf{r}, \mathbf{r}^{\prime}\right)+2 i k Z_{0} I \\
\times & \times\left[\frac{e^{i k R}}{4 \pi R}-\alpha \int_{0}^{\infty} e^{-\alpha \xi} \frac{e^{i k R^{\prime}}}{4 \pi R^{\prime}} d \xi\right]\left(l_{x} \hat{x}+l_{y} \hat{y}\right)-2 i Z_{0} I \eta l_{z} \hat{z} \\
\times & {\left[\left(i \frac{\partial}{\partial z}+\beta\right) \frac{e^{i k R}}{4 \pi R}+\left(k^{2}-\beta^{2}\right) \int_{0}^{\infty} e^{-\beta \xi} \frac{e^{i k R^{\prime}}}{4 \pi R^{\prime}} d \xi\right] } \\
+ & 2 Z_{0} I \eta \hat{l} \times\left(\frac{\partial}{\partial y} \hat{x}-\frac{\partial}{\partial x} \hat{y}\right) \\
\times & {\left[\frac{e^{i k R}}{4 \pi R}-\beta \int_{0}^{\infty} e^{-\beta \xi} \frac{e^{i k R^{\prime}}}{4 \pi R^{\prime}} d \xi\right]+\frac{2 i Z_{0} I \eta}{1-\eta^{2}} } \\
\times & \left\{\frac{\partial^{2}}{\partial x^{2}} l_{x} \hat{x}+\frac{\partial^{2}}{\partial y^{2}} l_{y} \hat{y}+\frac{\partial^{2}}{\partial x \partial y}\left(l_{y} \hat{x}+l_{x} \hat{y}\right)\right\} \int_{0}^{\infty} \\
& \cdot\left(e^{-\alpha \xi}-\eta^{2} e^{-\beta \xi}\right) \frac{e^{i k R^{\prime}}}{4 \pi R^{\prime}} d \xi
\end{aligned}
$$

where it is noted that the diverging exponential in the original expressions for the diffracted fields from a horizontal dipole, as well as the resulting static-image charge that appeared from this expression has vanished in the final formulation given.

\section{ANALYSIS AND RESULTS}

In this section, results generated by numerical evaluation of the exact image-integral expressions are given and compared to those generated by numerical evaluation of the original Sommerfeld-type expressions. Also, a comparison will be made of the computation time required to calculate field quantities using both methods. In the results that follow, all integrals are numerically evaluated using the Gaussian quadrature numerical integration package Quadpack, contained in the Slatec mathematical computation libraries. The Quadpack routines require defining both an absolute- and relative-error parameter and these were set at 0.0 and 0.001 , respectively.

For the initial comparison of the exact image formulation to the original Sommerfeld expression, consider an electric dipole located $2 \mathrm{~m}$ above the impedance surface $\left(z^{\prime}=2 \mathrm{~m}\right)$, at the coordinate origin $\left(x^{\prime}=y^{\prime}=0\right)$, and radiating at $30 \mathrm{MHz}$. All field quantities are normalized to dipole current, $I$ and wavelength, $\lambda(\mathbf{E} /(I / \lambda))$, for all cases. The geometry and coordinates are again shown in Fig. 1. The observation is on a radial line, $2 \mathrm{~m}$ above the impedance surface $(z=2 \mathrm{~m})$, ranging from $10 \mathrm{~m}$ to $10010 \mathrm{~m}$ along the $x$ axis $(\rho=10 \rightarrow 10010 \mathrm{~m}$, $\phi=0$ ), and field values are calculated at 11-data points along this line. The normalized surface impedance value is chosen to be $\eta=0.3-i 0.1$; corresponding to the impedance of San Antonio Gray Clay Loam with a 5\% gravimetric moisture content and a density of $1.4 \mathrm{~g} / \mathrm{cm}^{3}$, derived from the values of permittivity and conductivity given by Hipp [25].

Fig. 3 shows the $x$ component of the diffracted-electric field, for a vertical dipole, for this test case (no direct $x$ field component in this case). In Fig. 3, results from numerical evaluation of the exact image expressions are compared to results

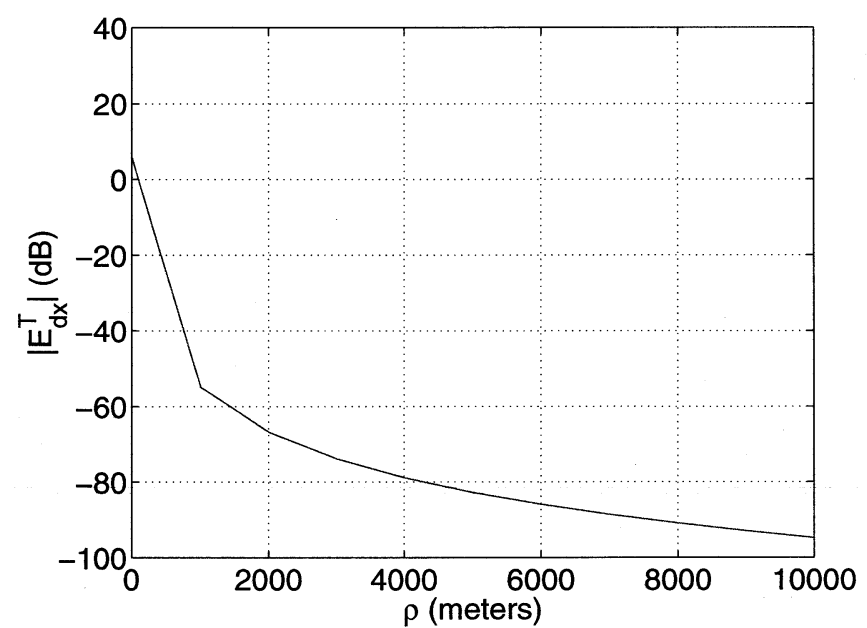

Fig. 3. $x$-component of total electric fields (diffracted only for this case) for a vertical $(\hat{z})$ electric dipole, exact image $(-)$ compared with Sommerfeld solution (- - -). Dipole is located at $\rho=0, z^{\prime}=2 \mathrm{~m}$ and operating at $30 \mathrm{MHz}$. Observation is at $z=2 \mathrm{~m}, \rho=10-10010 \mathrm{~m}$, along the $\phi=0$ ( $x$ axis). Normalized surface impedance value is $\eta=0.3-i 0.1$.

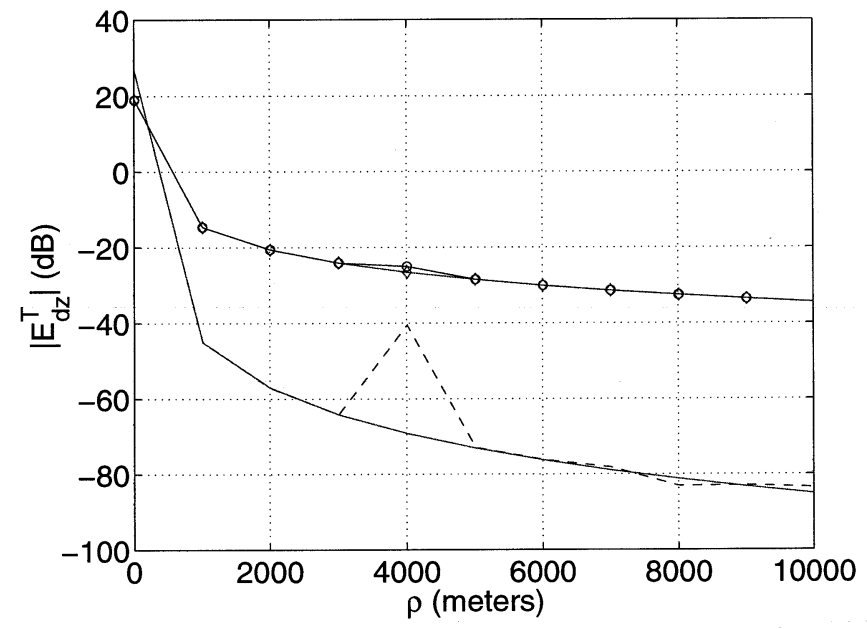

Fig. 4. $z$-component of diffracted [exact image $(\diamond \diamond \diamond \diamond \diamond)$ and Sommerfeld $(00000)$ ] and total electric fields [exact image ( - ) and Sommerfeld (- - - )] for a vertical $(\hat{z})$ electric dipole. Dipole is located at $\rho=0, z^{\prime}=2 \mathrm{~m}$ and operating at $30 \mathrm{MHz}$. Observation is at $z=2 \mathrm{~m}, \rho=10-10010 \mathrm{~m}$, along the $\phi=0$ ( $x$ axis). Normalized surface impedance value is $\eta=0.3-i 0.1$.

from numerical evaluation of the original Sommerfeld-type expressions. As can be seen, the two results are in excellent agreement. For the same test case, Fig. 4 shows the diffracted and total (direct + diffracted) $z$ electric field components for a vertical ( $z$ directed) dipole, again comparing the exact image calculation to those of the Sommerfeld formulation. As can be seen in Fig. 4, the diffracted fields are in good agreement, except for a slight discrepancy at $4000 \mathrm{~m}$, where the Sommerfeld calculation did not completely converge. The total fields in Fig. 4 show increased error at $4000 \mathrm{~m}$ for the Sommerfeld solution and also at distances beyond $6000 \mathrm{~m}$. This is due to the fact that the total field is the result of two large numbers (direct and diffracted field), tending to cancel for source and observation near the impedance surface. This has the effect of highlighting the numerical error in the Sommerfeld solution for the diffracted field, while the curves generated by the 


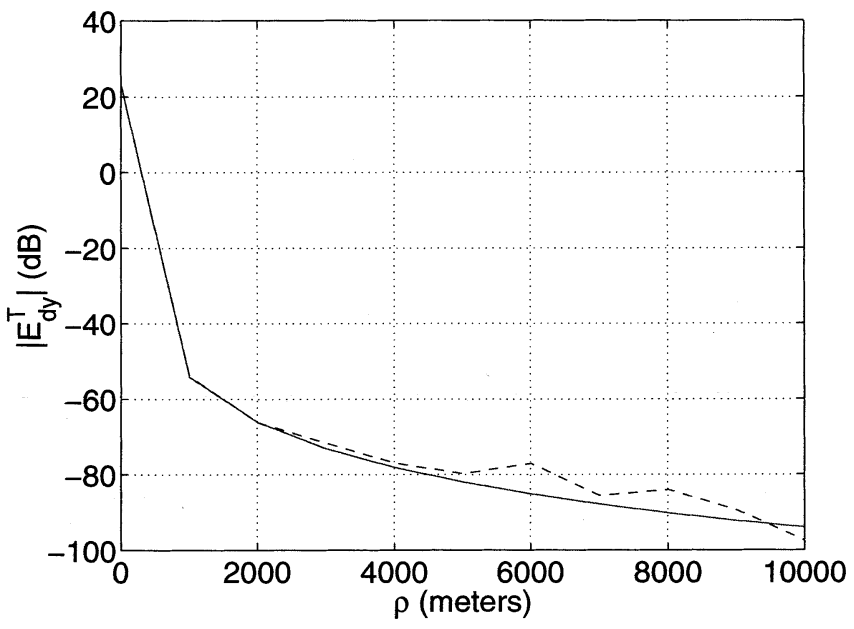

Fig. 5. $y$-component of total electric fields for a horizontal $(\hat{y})$ electric dipole, exact image ( - ) compared with Sommerfeld solution (- - -). Dipole is located at $\rho=0, z^{\prime}=2 \mathrm{~m}$ and operating at $30 \mathrm{MHz}$. Observation is at $z=2 \mathrm{~m}$, $\rho=10-10010 \mathrm{~m}$, along the $\phi=0$ ( $x$ axis). Normalized surface impedance value is $\eta=0.3-i 0.1$.

exact image formulation decay smoothly as expected, thus indicating better convergence in the numerical solution. This in fact is the case where higher-order terms (Norton surface wave) in the approximate asymptotic solutions dominate the total fields for $R \gg\left(z+z^{\prime}\right)$. The expression for $f_{c}$ given in (24), is simply the $z$ directed potential for a vertical dipole. Defining this potential as $U_{z}$, the asymptotic solution of $U_{z}$ can be decomposed as

$$
U_{z}=U_{z}^{i}+U_{z}^{\mathrm{go}}+U_{z}^{\mathrm{nsw}}
$$

where superscript $i$ indicates the direct wave, go is the GO term, equivalent to first-order saddle point, and $n s w$ indicates the Norton surface-wave component of the asymptotic solution, which is simply the higher-order terms in the saddle-point expansion. The first two terms in (50) are given by

$$
U_{z}^{i}+U_{z}^{g o}=\frac{e^{i k R_{0}}}{4 \pi R_{0}}+\Gamma_{v, s p} \frac{e^{i k R}}{4 \pi R}
$$

where $R_{0}=\sqrt{\left(x-x^{\prime}\right)^{2}+\left(y-y^{\prime}\right)^{2}+\left(z-z^{\prime}\right)^{2}}$ and $\Gamma_{v, s p}$ is the vertical Fresnel reflection coefficient evaluated at the saddle point, given by

$$
\Gamma_{v, s p}=\frac{\cos \theta-\eta}{\cos \theta+\eta} .
$$

In (52), $\cos \theta=\left(z+z^{\prime}\right) / R$ and $\theta$ is as defined in Fig. 1. Equation (51) can be rewritten as

$$
U_{z}^{i}+U_{z}^{\mathrm{go}}=\frac{e^{i k R_{0}}}{4 \pi R_{0}}-\frac{e^{i k R}}{4 \pi R}+\frac{2 \cos \theta}{\cos \theta+\eta} .
$$

As source and observation move near the impedance surface, the first two terms in (53) tend to cancel and the third term tends to zero as $R \rightarrow \infty, \theta \rightarrow \pi / 2$ and thus, higher-order terms in the asymptotic expansion (Norton surface wave) are dominant. These higher-order terms are given by

$$
U_{z}^{n s w}=\sqrt{\frac{i \pi}{2}} \frac{1}{k^{2} R^{2}}\left[\frac{1}{4} \Gamma_{v, s p}-\frac{1}{2}+\frac{4}{\eta^{2}}\right]
$$

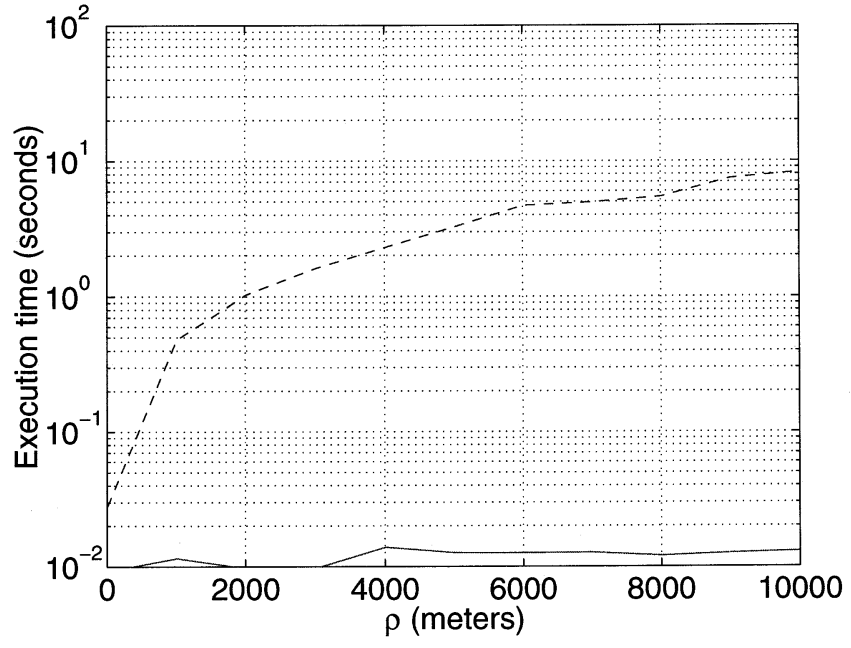

Fig. 6. Time (in seconds) to calculate all electric field components, at each observation point, for a vertical $(\hat{z})$ electric dipole, exact image $(-)$ and Sommerfeld solution (-- -). Dipole is located at $\rho=0, z^{\prime}=2 \mathrm{~m}$ and operating at $30 \mathrm{MHz}$. Observation is at $z=2 \mathrm{~m}, \rho=10-10010 \mathrm{~m}$, along the $\phi=0$ ( $x$ axis). Normalized surface impedance value is $\eta=0.3-i 0.1$.

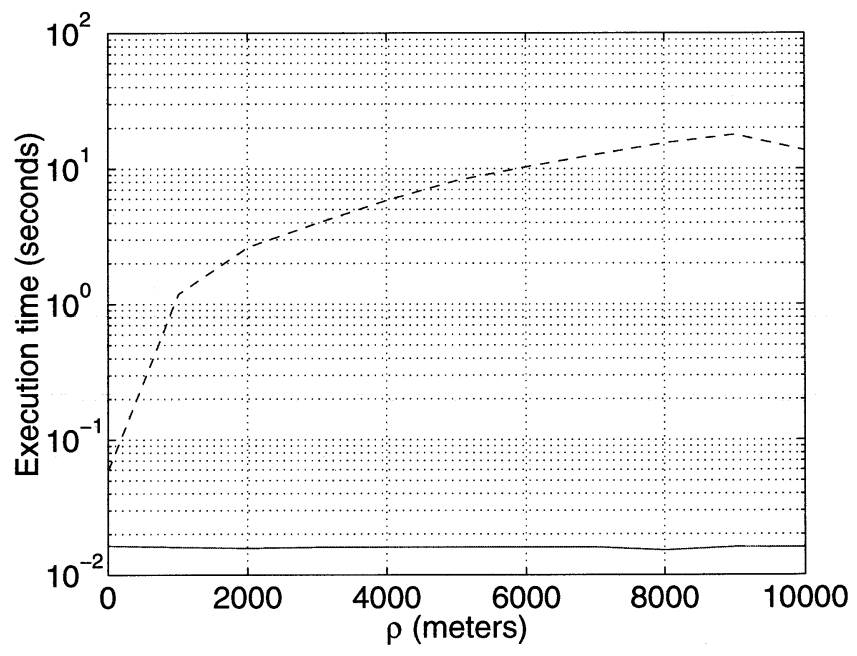

Fig. 7. Time (in seconds) to calculate all electric field components, at each observation point, for a horizontal $(\hat{y})$ electric dipole, exact image $(-)$ and Sommerfeld solution (- - -). Dipole is located at $\rho=0, z^{\prime}=2 \mathrm{~m}$ and operating at $30 \mathrm{MHz}$. Observation is at $z=2 \mathrm{~m}, \rho=10-10010 \mathrm{~m}$, along the $\phi=0$ ( $x$ axis). Normalized surface impedance value is $\eta=0.3-i 0.1$.

where in (54), it is assumed that $|\eta| \gg \cos \theta$ and that any pole is not in the vicinity of the saddle point. Fig. 5 shows the $y$ components of the total electric field for a horizontal ( $y$ directed) dipole and for the same test case. Again, the convergence problems of the Sommerfeld integral are apparent in Fig. 5 in the degradation of the appropriate curve beyond 2000 m. In Figs. 6 and 7 , the computation time (in seconds), required to calculate all field components at each observation point is plotted for this test case. The curves in Fig. 6 compare the time required for numerical evaluation of the Sommerfeld-type solution for the case of a vertical dipole, to the time required for the exact image formulation to perform the same field calculations. Fig. 7 shows a similar comparison for a horizontal ( $y$ directed) electric dipole. As is obvious from both sets of curves, the computation time required for the exact image calculations are significantly faster than the time required to calculate the 
Sommerfeld integrals, in fact over two orders of magnitude faster as the observation distance goes beyond $2000 \mathrm{~m}$. Note that for both methods, only necessary integrals were evaluated, for example, the integrals containing $l_{x}$ were not called if the dipole was strictly $z$ directed ( $l_{z}$ component only). In continuing with a comparison of computation time between exact image and the Sommerfeld integrals, Table I shows a comparison of the speed-up in computation time required by the exact image formulation over the original Sommerfeld-type expressions. Speed-up is defined as the ratio of the time required to calculate the Sommerfeld expressions to that required to perform the exact-image calculations [Sommerfeld time (seconds)/exact image time (seconds)]. In Table I, the computation time for each method is the time required to calculate all field components at all observation positions (still eleven data points, from $\rho=10 \rightarrow$ $10010 \mathrm{~m}, \phi=0$ ), again for the case of $\eta=0.3-i 0.1$, but with varying source and observation heights. As seen in Table I, the exact image calculations exhibit a significant speed-up in convergence time over the Sommerfeld-type expression for numerical evaluation of the integrals. As a final comparison of computation times, Table II shows the speed up in computation time of the exact image formulation over the Sommerfeld-type expressions, for varying normalized complex impedance values, again for 11 data points, from $\rho=10 \rightarrow 10010 \mathrm{~m}, \phi=0$, and for source and observation $2 \mathrm{~m}$ above the impedance surface. Again, the exact image calculations are at least an order of magnitude faster than the Sommerfeld calculations for all cases except that of a (PEC).

Also in this section, the effects of varying soil moisture on the total electric fields of a vertical dipole, are presented. The dipole is again radiating at $30 \mathrm{MHz}$, with source $2 \mathrm{~m}$ above the impedance surface, observation also $2 \mathrm{~m}$ above the impedance surface, and again along the $x$ axis from $\rho=10$ to $\rho=10010 \mathrm{~m}$. Fig. 8 shows a comparison of the $z$ component of the total electric fields for the same San Antonio Gray Clay Loam previously described, again with a density of $1.4 \mathrm{~g} / \mathrm{cm}^{3}$. The curves show field levels for gravimetric moisture contents of $0 \%, 2.5 \%, 5 \%, 10 \%$, and $20 \%$, corresponding to normalized surface impedances $(\eta)$ of $0.53,0.38-i 0.09,0.3-i 0.1$, $0.15-i 0.09$, and $0.12-i 0.07$, respectively. As can be seen in Fig. 8, the effect of increasing moisture content is to increase the vertical component of the total electric fields by as much as $20 \mathrm{~dB}$ over the range shown. A similar analysis for a horizontal dipole ( $y$ directed and again observation along the $x$ axis) show the total field levels to be essentially insensitive to varying soil moisture.

As a final example, the frequency response of the field of a vertical dipole over the impedance half-space is examined. The frequency response is indicative of the dispersive effects of the half-space and these effects are of significant interest in the point to point transmission of wide-band radio signals over the earth. For this example, the source and observation are placed $2 \mathrm{~m}$ above the surface and the frequency response is examined at radial distance $\rho=300 \mathrm{~m}$ from the source. Frequency is swept from 30 to $130 \mathrm{MHz}$ in steps of $142.86 \mathrm{KHz}$. For obvious reasons, the electric field quantities are not normalized to $I / \lambda$ as in the previous examples. Also, field expressions must be multiplied by the dipole length (in meters) and in this example,
TABLE I

SPEED-UP IN COMPUTATION TIME OF EXACT IMAGE CALCULATION OVER SOMMERFELD FORMULATION FOR NORMALIZED SURFACE IMPEDANCE OF $0.3-i 0.1$, ElEVEN DATA POINTS FROM $\rho=10 \rightarrow 10010, \phi=0$ AND VARYING SOURCE/OBSERVATION HEIGHTS

\begin{tabular}{c|c|c|c}
\hline$z^{\prime}(m)$ & $z(m)$ & Speed-up, Vertical Dipole & Speed-up, Horizontal Dipole \\
\hline 2 & 2 & 303.92 & 565.96 \\
\hline 2 & 200 & 54.89 & 106.92 \\
\hline 200 & 2 & 56.11 & 107.00 \\
\hline 200 & 200 & 65.00 & 110.00 \\
\hline
\end{tabular}

TABLE II

SPEED-Up IN COMPUTATION TIME OF EXACT IMAge CALCULATION OVER SOMMERFELD FORMULATION FOR VARYING COMPLEX IMPEDANCE, $\eta$. SOURCE AND ReCEIVER ARE $2 \mathrm{~m}$ Above SuRfaCe FOR aLl CASES. EleVEn Data Points ARe Calculated From $\rho=10 \rightarrow 10010, \phi=0$

\begin{tabular}{c|c|c}
\hline$\eta$ & Speed-up, Vertical Dipole & Speed-up, Horizontal Dipole \\
\hline $0.0-\mathrm{i} 0.0$ & 5.41 & 13.86 \\
\hline $0.1-\mathrm{i} 0.0$ & 282.00 & 613.80 \\
\hline $0.3-\mathrm{i} 0.0$ & 318.17 & 624.60 \\
\hline $0.5-\mathrm{i} 0.0$ & 490.38 & 715.92 \\
\hline $0.003-\mathrm{i} 0.1$ & 9.81 & 31.03 \\
\hline $0.003-\mathrm{i} 0.3$ & 10.03 & 18.3 \\
\hline $0.003-\mathrm{i} 0.5$ & 9.96 & 14.51 \\
\hline $0.1-\mathrm{i} 0.1$ & 254.29 & 425.62 \\
\hline $0.3-\mathrm{i} 0.3$ & 266.00 & 452.20 \\
\hline $0.5-\mathrm{i} 0.5$ & 348.25 & 510.28 \\
\hline
\end{tabular}

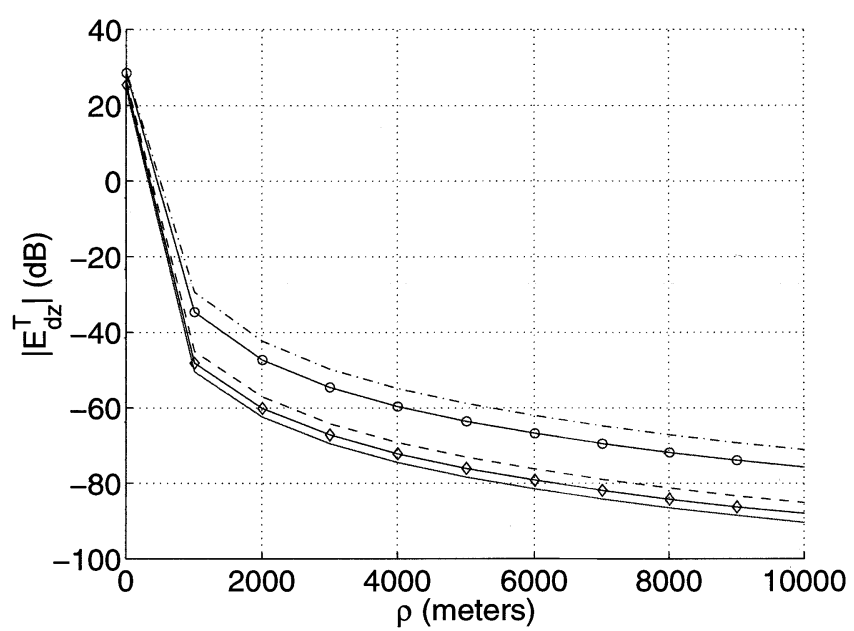

Fig. 8. Effect of varying soil moisture on $z$-component of total electric fields for a vertical $(\hat{z})$ dipole located at the origin, $2 \mathrm{~m}$ above an impedance surface, $\left(\rho=0, z^{\prime}=2 \mathrm{~m}\right)$ and operating at $30 \mathrm{MHz}$. Observation is also $2 \mathrm{~m}$ above the surface and extends radially from the source along the $\phi=0$ ( $x$ axis) from $\rho=10-10010 \mathrm{~m}$. Results are for soil moisture of $0 \%(\eta=0.53,(-))$, $2.5 \%(\eta=0.38-i 0.09,(\diamond \diamond \diamond \diamond \diamond)), 5 \%(\eta=0.3-i 0.1,(---)), 10 \%$ $(\eta=0.15-i 0.09,(00000))$ and $20 \%(\eta=0.12-i 0.07,(\cdot-\cdot-\cdot-\cdot))$.

the length is set at $0.2307 \mathrm{~m}(\lambda / 10$ at $130 \mathrm{MHz})$. As the normalized impedance of the surface varies with frequency, the appropriate real component of the relative permittivity $\left(\varepsilon^{\prime}\right)$ and conductivity $(\sigma)$ are selected from the tables given by Hipp [25] and the normalized impedance calculated from these parameters at each frequency. As these values are slowing, varying functions of frequency, values at the center of the frequency band $(80 \mathrm{MHz})$ are chosen and assumed to be constant across the band. The normalized impedance, $\eta$, at each frequency is then given by $\eta=1 / \sqrt{\varepsilon^{\prime}+i \varepsilon^{\prime \prime}}$, where 


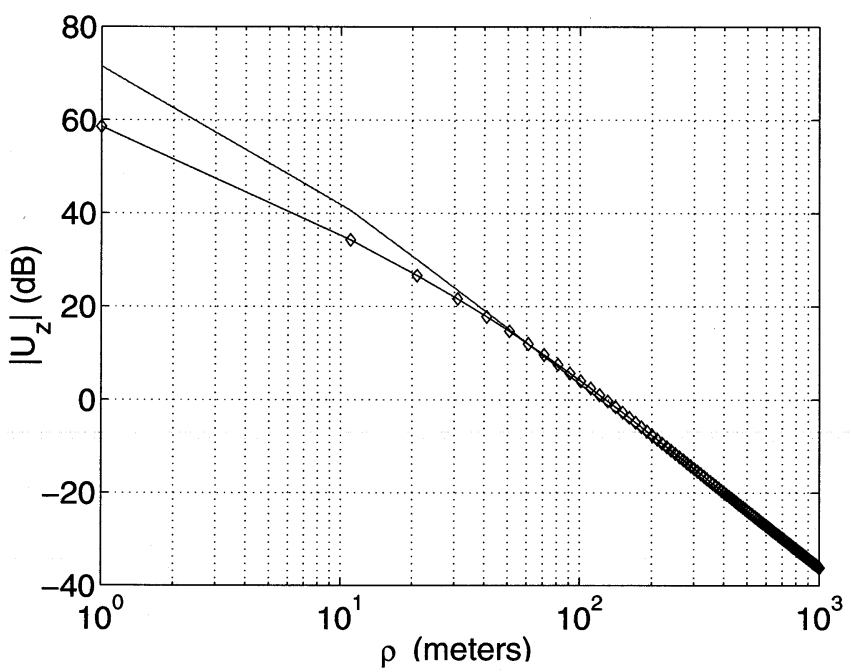

Fig. 9. Comparison of potentials from asymptotic solution and exact-image theory for a vertical $(\hat{z})$ dipole located at the origin, $0.1 \mathrm{~m}$ above the surface $\left(\rho=0, z^{\prime}=0.1 \mathrm{~m}\right)$ with normalized impedance $\eta=0.3-i 0.1$ and operating at $30 \mathrm{MHz}$. Observation is also $0.1 \mathrm{~m}$ above the surface and extends radially from the source along the $\phi=0$ ( $x$ axis) from $\rho=1-1001 \mathrm{~m}$. Second-order saddle point $(-)$ compared with exact image $(\diamond \diamond \diamond \diamond \diamond \diamond)$.

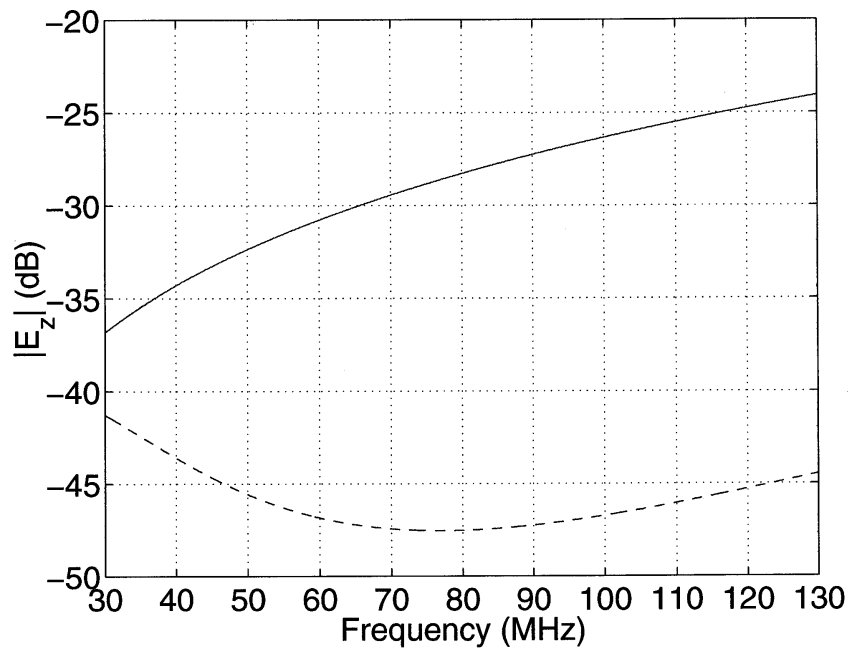

Fig. 10. Comparison of the magnitude of the frequency response of the direct field (- ${ }^{-}$) to total field (- - - ), for a vertical $(\hat{z})$ dipole located at the origin, $2.0 \mathrm{~m}$ above the impedance surface $\left(\rho=0, z^{\prime}=2.0 \mathrm{~m}\right)$ with real permittivity $\varepsilon^{\prime}=22.0$, conductivity $\sigma=8 \times 10^{-2}$. Frequency sweep is from 30 to 130 $\mathrm{MHz}$ in steps of $142.86 \mathrm{KHz}$. Observation is also $2.0 \mathrm{~m}$ above the surface along the $\phi=0(x$ axis $)$ and $300 \mathrm{~m}$ from the source $(\rho=300 \mathrm{~m})$.

$\varepsilon^{\prime \prime}=\sigma /\left(\omega \varepsilon_{0}\right)$ and $\varepsilon_{0}=8.85 \times 10^{-12}$ is the permittivity of free space. For this example, these values were chosen to be $\varepsilon^{\prime}=22.0$ and $\sigma=8 \times 10^{-2}$, which correspond to values for San Antonio Gray Clay Loam with a $20 \%$ gravimetric moisture content and a density of $1.4 \mathrm{~g} / \mathrm{cm}^{3}$. Figs. 10 and 11 compare the magnitude and phase of the frequency response of the direct dipole field to that of the total field. It is shown that the magnitude of the frequency response for the total field is monotonic and slowing varying while the phase is essentially linear and almost identical to the phase of the direct field. The phase behavior indicates that there is little or no dispersion of the broad-band signal in such communications channels. This was found to be true for various complex impedances

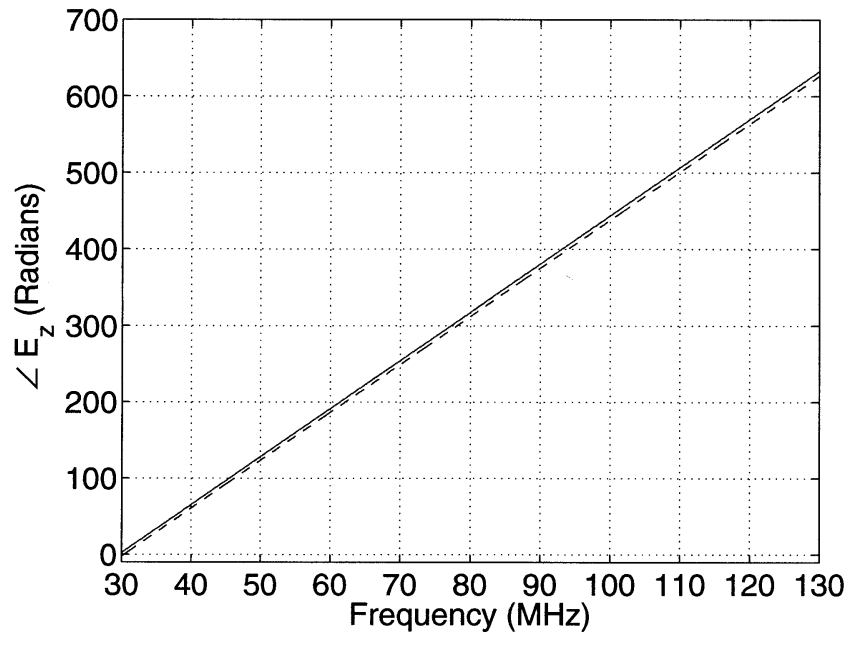

Fig. 11. Comparison of the phase of the frequency response of the direct field ( $\longrightarrow$ ) to total field (- - - ) (in radians), for a vertical $(\hat{z})$ dipole located at the origin, $2.0 \mathrm{~m}$ above the impedance surface $\left(\rho=0, z^{\prime}=2.0 \mathrm{~m}\right)$ with real permittivity $\varepsilon^{\prime}=22.0$, conductivity $\sigma=8 \times 10^{-2}$. Frequency sweep is from 30 to $130 \mathrm{MHz}$ in steps of 142.86 . Observation is also $2.0 \mathrm{~m}$ above the surface along the $\phi=0$ ( $x$ axis) and $300 \mathrm{~m}$ from the source $(\rho=300 \mathrm{~m})$.

corresponding to different moisture content, with the exception of the case of either source or observation raised significantly above the surface where there is significant difference in path delay between the direct and ground (diffracted) waves.

\section{SUMmary AND CONCLUSION}

In this paper, exact image theory is applied to improve the convergence properties of the Sommerfeld-type integrals contained in the spectral domain representation of the fields of an infinitesimal electric dipole above an impedance surface. The Sommerfeld expressions are written in terms of zeroth-order Bessel functions and the reflection coefficients in the form of a simple Laplace transform. Where necessary, terms are expanded by partial fractions so they can be expressed in this form. Order of integration is then exchanged and the inverse transform from the spectral domain is performed analytically. The remaining expressions consist of integrals whose integrand is dominated by a decaying exponential and exhibits rapid convergence qualities. Numerical evaluation of these integrals show good agreement with results obtained by numerical evaluation of the Sommerfeld-type integrals, while exhibiting a speed up in the computation time of several orders of magnitude.

In conclusion, a brief discussion is appropriate on the applications of this exact image formulation to problems of practical interest. For obstacles of moderate electric size, IE techniques can be applied, however these techniques require evaluation of the Green's function of the problem. As is well known, the Sommerfeld solution for the fields of an infinitesimal dipole radiating above an impedance surface is, except for some constant coefficients, the Green's function of the impedance half-space. Numerical evaluation of this Green's function is impractical due to the prohibitive computational time required to evaluate the highly oscillatory Sommerfeld integrals for each element of the IE matrix solution. IE techniques require accurate evaluation of the Green's function for all source and observation positions and, therefore, approximate solutions to 
the Sommerfeld integrals also cannot be applied. As an example of this, Fig. 9 shows the $z$ directed potential for a vertical dipole generated by exact-image theory, compared to the potential generated by evaluating the Sommerfeld integral in an approximate manner using second-order saddle point. As can be seen in Fig. 9, the expected degradation in the accuracy of the saddle-point technique is observed as the observation position approaches the source and the assumption of the large distance from source to observation point is violated, thus making it of no practical use in IE applications. Note that for these curves, the normalized surface impedance was $\eta=0.3-i 0.1$ and source and observation were placed $0.1 \mathrm{~m}$ above the surface as might be found in a typical IE formulation. Also, for this surface impedance value, the pole is isolated from the saddle point for all observation positions, thus making standard saddle-point techniques applicable [10]. Because of the discussed limitations, application of the Sommerfeld solution to IE techniques has been impractical. The significant improvement in the convergence properties of the Sommerfeld solution by application of the exact-image transforms, and the resulting speed up in the computation time now makes the Sommerfeld solution of practical use in IE applications.

\section{APPENDIX}

SPECTRAL REPRESENTATION OF THE FIELDS OF A SMALL DIPOLE OF ARBITRARY ORIENTATION, RADIATING ABOVE AN INFINITE IMPEDANCE SURFACE

In this section, the general formulation for the electric fields of an infinitesimal electric dipole radiating above a homogeneous, infinite impedance surface is provided. The geometry of the problem is as shown in Fig. 1. First, the spectral domain representation of the dipole fields is given. A change of variables is then applied and appropriate application of Bessel identities will result in the final form of the dipole fields.

The spectral-domain representation for the total-electric fields of a small-electric dipole radiating above an impedance half-space, with orientation $\hat{l}$ and carrying current $I$, is given by [26], as shown in (A.1) at the bottom of the next page. In (A.1), $\mathbf{k}=k_{x} \hat{x}+k_{y} \hat{y}+k_{z} \hat{z}$ with dependent variable, $k_{z}$ defined as $k_{z}=\sqrt{k^{2}-k_{x}^{2}-k_{y}^{2}}$, and the branch cut defined as $i=\sqrt{-1}$. The polarization unit vectors $\hat{h}$ and $\hat{v}$ are given by

$$
\hat{h}\left(k_{z}\right)=\frac{\mathbf{k} \times \hat{z}}{|\mathbf{k} \times \hat{z}|} \quad \hat{v}\left(k_{z}\right)=\frac{\hat{h}\left(k_{z}\right) \times \mathbf{k}}{k}
$$

and

$$
\hat{h}\left(-k_{z}\right)=\frac{\mathbf{K} \times \hat{z}}{|\mathbf{K} \times \hat{z}|} \quad \hat{v}\left(-k_{z}\right)=\frac{\hat{h}\left(-k_{z}\right) \times \mathbf{K}}{k}
$$

where $\hat{h}$ indicates horizontal polarization [transverse electric (TE) to $z$ ] and $\hat{v}$ indicates vertical polarization [transverse magnetic (TM) to $z$ ], and $\mathbf{K}=\mathbf{k}-2(\mathbf{k} \cdot \hat{z}) \hat{z}=k_{x} \hat{x}+k_{y} \hat{y}-k_{z} \hat{z}$. The reflection coefficients $\Gamma_{h}$ and $\Gamma_{v}$ (horizontal and vertical reflection coefficients, respectively) in (A.1) are defined as

$$
\Gamma_{h}=\frac{\eta-k / k_{z}}{\eta+k / k_{z}} \quad \Gamma_{v}=\frac{-\eta+k_{z} / k}{\eta+k_{z} / k}
$$

where $\eta$ is the normalized surface impedance, $\eta=Z_{1} / Z_{0}$. The terms containing $\Gamma_{h}$ and $\Gamma_{v}$, to the left of the + signs in (A.1) represent the effects of the impedance surface on the total field and are designated as the diffracted fields, with those to the right of the + sign representing the direct dipole fields. The direct dipole electric fields are more conveniently evaluated in the spatial domain and are given in dyadic form by

$$
\mathbf{E}_{d}^{i}\left(\mathbf{r}, \mathbf{r}^{\prime}\right)=-i k Z_{0} I\left[\mathbf{I}+\frac{1}{k^{2}} \nabla \nabla\right] \frac{e^{i k\left|\mathbf{r}-\mathbf{r}^{\prime}\right|}}{4 \pi R_{0}} \cdot \hat{l}
$$

where $|\mathbf{r}|=\sqrt{x^{2}+y^{2}+z^{2}}$ is the distance to the observation point and $\left|\mathbf{r}^{\prime}\right|=\sqrt{x^{\prime 2}+y^{\prime 2}+z^{\prime 2}}$ is the distance to the source location. Expanding (A.5) gives the more useful form of the direct dipole fields or

$$
\begin{array}{r}
\mathbf{E}^{i}\left(\mathbf{r}, \mathbf{r}^{\prime}\right)=i k Z_{0} I\{( \\
\left.k^{2} R_{0}^{2}-\frac{3 i}{k R_{0}}-1\right) \hat{R}_{0}\left(\hat{l} \cdot \hat{R}_{0}\right) \\
\left.+\left(1+\frac{i}{k R_{0}}-\frac{1}{k^{2} R_{0}^{2}}\right) \hat{l}\right\} \frac{e^{i k R_{0}}}{4 \pi R_{0}}
\end{array}
$$

where

$$
R_{0}=\left|\mathbf{r}-\mathbf{r}^{\prime}\right|=\sqrt{\left(x-x^{\prime}\right)^{2}+\left(y-y^{\prime}\right)^{2}+\left(z-z^{\prime}\right)^{2}}
$$

and

$$
\hat{R}_{0}=\frac{\mathbf{r}-\mathbf{r}^{\prime}}{\left|\mathbf{r}-\mathbf{r}^{\prime}\right|}
$$

To derive the Sommerfeld expressions for the diffracted electric fields above the impedance surface we first apply the standard change of variables to (A.1)

$$
\begin{array}{ll}
k_{x}=k_{\rho} \cos \nu & x-x^{\prime}=\rho \cos \phi \\
k_{y}=k_{\rho} \sin \nu & y-y^{\prime}=\rho \sin \phi
\end{array}
$$

resulting in the following expression for the diffracted dipole fields

$$
\begin{aligned}
\mathbf{E}^{d}\left(\mathbf{r}, \mathbf{r}^{\prime}\right)= & \frac{k Z_{0} I}{8 \pi^{2}} \int_{0}^{2 \pi} \int_{0}^{\infty} \frac{k_{\rho}}{k_{z}} \\
& \cdot\left[\Gamma_{h}(\hat{h} \cdot \hat{l}) \hat{h}+\Gamma_{v}\left(\hat{v}\left(-k_{z}\right) \cdot \hat{l}\right) \hat{v}\left(k_{z}\right)\right] \\
& \cdot e^{i k_{z}\left(z+z^{\prime}\right)} e^{i k_{\rho} \rho \cos (\nu-\phi)} d \nu d k_{\rho}
\end{aligned}
$$

In (A.8), $(\hat{h} \cdot \hat{l}) \hat{h}$ and $\left(\hat{v}\left(-k_{z}\right) \cdot \hat{l}\right) \hat{v}\left(k_{z}\right)$ can be rewritten in terms of the new variables as

$$
\begin{aligned}
(\hat{h} \cdot \hat{l}) \hat{h}=\left(l_{x} \sin ^{2} \nu-\right. & \left.l_{y} \sin \nu \cos \nu\right) \hat{x} \\
& +\left(-l_{x} \sin \nu \cos \nu+l y \cos ^{2} \nu\right) \hat{y}
\end{aligned}
$$

and

$$
\begin{aligned}
\left(\hat{v}\left(-k_{z}\right) \cdot \hat{l}\right) \hat{v}\left(k_{z}\right) & \\
=\frac{1}{k^{2}}\{ & -k_{z} \cos \nu\left[k_{\rho} l_{z}+k_{z}\left(l_{x} \cos \nu+l_{y} \sin \nu\right] \hat{x}\right. \\
& \quad-k_{z} \sin \nu\left[k_{\rho} l_{z}+k_{z}\left(l_{x} \cos \nu+l_{y} \sin \nu\right)\right] \hat{y} \\
& \left.+k_{\rho}\left[k_{\rho} l_{z}+k_{z}\left(l_{x} \cos \nu+l_{y} \sin \nu\right)\right] \hat{z}\right\} .
\end{aligned}
$$

Substituting (A.9) and (A.10) into (A.8) and recognizing that $\cos ^{2} \nu=(1+\cos 2 \nu) / 2, \sin ^{2} \nu=(1-\cos 2 \nu) / 2$, and 
$\sin \nu \cos \nu=(1 / 2) \sin 2 \nu$ and by applying the following two identities

$$
\begin{aligned}
& \int_{0}^{2 \pi} \cos (n \nu) e^{i k_{\rho} \rho \cos (\nu-\phi)} d \nu=2 \pi(i)^{n} \cos (n \phi) J_{n}\left(k_{\rho} \rho\right) \\
& \int_{0}^{2 \pi} \sin (n \nu) e^{i k_{\rho} \rho \cos (\nu-\phi)} d \nu=2 \pi(i)^{n} \sin (n \phi) J_{n}\left(k_{\rho} \rho\right)
\end{aligned}
$$

the diffracted electric fields in (A.8) can be rewritten as

$$
\begin{aligned}
\mathbf{E}^{d}(\mathbf{r}, & \left.\mathbf{r}^{\prime}\right) \\
= & \frac{k Z_{0} I}{4 \pi}\left\{\hat { x } \int _ { 0 } ^ { \infty } \frac { k _ { \rho } } { 2 k _ { z } } \left\{\Gamma _ { h } \left[-l_{x}\left(J_{2}\left(k_{\rho} \rho\right) \cos 2 \phi\right.\right.\right.\right. \\
& \left.\left.\left.+J_{0}\left(k_{\rho} \rho\right)\right)-l_{y} J_{2}\left(k_{\rho} \rho\right) \sin 2 \phi\right)\right] \\
& +\Gamma_{v}\left[\frac{2 i k_{z} k_{\rho}}{k^{2}} l_{z} \cos \phi J_{1}\left(k_{\rho} \rho\right)+\frac{k_{z}^{2}}{k^{2}} l_{x}\right. \\
& +\left(J_{0}\left(k_{\rho} \rho\right)-J_{2}\left(k_{\rho} \rho\right) \cos 2 \phi\right) \\
& \left.\left.-\frac{k_{z}^{2}}{k^{2}} l_{y} J_{2}\left(k_{\rho} \rho\right) \sin 2 \phi\right]\right\} e^{i k_{z}\left(z+z^{\prime}\right)} d k_{\rho} \\
& +\hat{y} \int_{0}^{\infty} \frac{k_{\rho}}{2 k_{z}}\left\{\Gamma _ { h } \left[-l_{x} J_{2}\left(k_{\rho} \rho\right) \sin 2 \phi\right.\right. \\
& \left.-l_{y}\left(J_{0}\left(k_{\rho} \rho\right)-J_{2}\left(k_{\rho} \rho\right) \cos 2 \phi\right)\right] \\
& +\Gamma_{v}\left[\frac{2 i k_{z} k_{\rho}}{k^{2}} l_{z} \sin \phi J_{1}\left(k_{\rho} \rho\right)-\frac{k_{z}^{2}}{k^{2}} l_{x} J_{2}\left(k_{\rho} \rho\right) \sin 2 \phi\right. \\
& \left.\left.+\frac{k_{z}^{2}}{k^{2}} l_{y}\left(J_{0}\left(k_{\rho} \rho\right)+J_{2}\left(k_{\rho} \rho\right) \cos 2 \phi\right)\right]\right\} e^{i k_{z}\left(z+z^{\prime}\right)} d k_{\rho} \\
& -\hat{z} \int_{0}^{\infty} \frac{k_{\rho}}{k_{z}} \Gamma_{v}\left[\frac{k_{\rho}^{2}}{k^{2}} l_{z} J_{0}\left(k_{\rho} \rho\right)+\frac{i k_{z} k_{\rho}}{k^{2}}\right. \\
& \left.\left.+\left(l x \cos \phi+l_{y} \sin \phi\right) J_{1}\left(k_{\rho} \rho\right)\right] e^{i k_{z}\left(z+z^{\prime}\right)} d k_{\rho}\right\} .(\mathrm{A} .13
\end{aligned}
$$

The expressions contained in (A.13) are of the form derived by Sommerfeld and the integrals contained within are defined as Sommerfeld type integrals.

\section{REFERENCES}

[1] K. Sarabandi and M. D. Casciato, "Diffraction of radio waves from arbitrary one-dimensional surface impedance discontinuities," IEEE Trans. Antennas Propagat., vol. 47, pp. 86-96, Jan. 1999.

[2] A. Sommerfeld, "Über die Ausbreitung der Wellen in der drahtlosen Telegraphie," Ann. Physik, vol. 28, pp. 665-736, 1909.

[3] H. Weyl, "Ausbreitung elektromagnetischer wellen über einem ebenen Leiter," Ann. Physik, vol. 60, pp. 481-500, 1919.

[4] A. Sommerfeld, "Über die Ausbreitung der Wellen in der drahtlosen Telegraphie," Ann. Physik, vol. 81, pp. 1135-1153, 1926.

[5] B. van der Pol and K. F. Niessen, "Über die Ausbreitung elektromagnetischer Wellen über eine ebene Erde," Ann. Physik, vol. 6, pp. 273-294, 1930.

[6] W. H. Wise, "The grounded condenser antenna radiation formula," Proc. I.R.E., vol. 19, pp. 1684-1689, Sept. 1931.

[7] K. A. Norton, "The propagation of radio waves over the surface of the Earth and in the upper atmosphere: Part I," Proc. IRE, vol. 24, no. 10, pp. 1367-1387, Oct. 1936.

[8] — "The propagation of radio waves over the surface of the Earth and in the upper atmosphere: Part II," Proc. I.R.E., vol. 25, no. 9, pp. 1203-1236, Sept. 1937.

[9] A. Baños, Dipole Radiation in the Presence of a Conducting Half-Space. New York: Pergamon, 1966.

[10] L. B. Felsen and N. Marcuvitz, Radiation and Scattering of Waves. Piscataway, NJ: IEEE Press, 1994.

[11] J. R. Wait, Electromagnetic Waves in Stratified Media. Piscataway, NJ: IEEE Press, 1996.

[12] W. C. Chew, Waves and Fields in Inhomogeneous Media. New York: Van Nostrand, 1990.

[13] P. Parhami, Y. Rahmat-Samii, and R. Mittra, "An efficient approach for evaluating Sommerfeld integrals encountered in the problem of a current element radiating over lossy ground," IEEE Trans. Antennas Propagat., vol. 28, pp. 100-104, Jan. 1980.

[14] K. A. Michalski, "On the efficient evaluation of integrals arising in the Sommerfeld half-space problem," Inst. Elect. Eng. Proc. Part $\mathrm{H}-\mathrm{Mi}$ crowave, Antennas Propagat., vol. 132, no. 5, pp. 312-318, Aug. 1985.

[15] W. A. Johnson and D. G. Dudley, "Real axis integration of Sommerfeld integrals: Source and observation points in air," Radio Sci., vol. 18, no. 2, pp. 313-324, Mar.-Apr. 1983.

[16] B. van der Pol, "Theory of the reflection of the light from a point source by a finitely conducting flat mirror: With an application to radiotelegraphy," Physica, vol. 2, pp. 843-853, 1935.

[17] K. Furutsu, "On the excitation of the waves of proper solutions," I.R.E. Trans. Antennas Propagat., vol. 7, pp. 209-218, 1959. Special Supplement.

[18] A. N. Sommerfeld, Partial Differential Equations in Physics. New York: Academic, 1949.

[19] H. G. Booker and P. C. Clemmow, "A relation between the Sommerfeld theory of radio wave propagation over a flat Earth and the theory of diffraction at a straight edge," Proc. Inst. Elect. Eng. , pt. III, vol. 97, no. 45, pp. 18-27, Jan. 1950.

$\mathbf{E}^{T}\left(\mathbf{r}, \mathbf{r}^{\prime}\right)=-\frac{i I Z_{0}}{k}(\hat{l} \cdot \hat{z}) \hat{z} \delta\left(\mathbf{r}-\mathbf{r}^{\prime}\right)$

$$
+\left\{\begin{array}{cc}
-\frac{k Z_{0} I}{8 \pi^{2}} \int_{-\infty}^{+\infty} d k_{x} d k_{y} \frac{e^{i\left(k_{x}\left(x-x^{\prime}\right)+k_{y}\left(y-y^{\prime}\right)\right)}}{k_{z}} & \\
\left\{\hat{h}\left(k_{z}\right)\left[\Gamma_{h}\left(\hat{h}\left(k_{z}\right) \cdot \hat{l}\right) e^{i k_{z} z^{\prime}}+\left(\hat{h}\left(k_{z}\right) \cdot \hat{l}\right) e^{-i k_{z} z^{\prime}}\right]\right. & \\
\left.+\hat{v}\left(k_{z}\right)\left[\Gamma_{v}\left(\hat{v}\left(-k_{z}\right) \cdot \hat{l}\right) e^{i k_{z} z^{\prime}}+\left(\hat{v}\left(k_{z}\right) \cdot \hat{l}\right) e^{-i k_{z} z^{\prime}}\right]\right\} e^{i k_{z} z} & \text { for } \mathbf{z}>\mathbf{z}^{\prime} \\
-\frac{k Z_{0} I}{8 \pi^{2}} \int_{-\infty}^{+\infty} d k_{x} d k_{y} \frac{e^{i\left(k_{x}\left(x-x^{\prime}\right)+k_{y}\left(y-y^{\prime}\right)\right)}}{k_{z}} & \\
\left\{\left[\hat{h}\left(k_{z}\right) \Gamma_{h} e^{i k_{z} z}+\hat{h}\left(k_{z}\right) e^{-i k_{z} z}\right]\left(\hat{h}\left(k_{z}\right) \cdot \hat{l}\right)\right. & \\
\left.+\left[\hat{v}\left(k_{z}\right) \Gamma_{v} e^{i k_{z} z}+\hat{v}\left(-k_{z}\right) e^{-i k_{z} z}\right]\left(\hat{v}\left(-k_{z}\right) \cdot \hat{l}\right)\right\} e^{i k_{z} z^{\prime}} & \text { for } \mathbf{0}<\mathbf{z}<\mathbf{z}^{\prime} .
\end{array}\right.
$$


[20] J. B. Keller, "Oblique derivative boundary conditions and the image method," SIAM J. Appl. Math., vol. 41, pp. 294-300, 1981

[21] I. V. Lindell and E. Alanen, "Exact image theory for the Sommerfeld half-space problem, Part I: Vertical magnetic dipole," IEEE Trans. Antennas Propagat., vol. AP-32, pp. 126-133, Feb. 1984.

[22] — "Exact image theory for the Sommerfeld half-space problem, Part II: Vertical electric dipole," IEEE Trans. Antennas Propagat., vol. AP-32, pp. 841-847, Aug. 1984.

[23] _ "Exact image theory for the Sommerfeld half-space problem, Part III: General formulation," IEEE Trans. Antennas Propagat., vol. AP-32, pp. 1027-1032, Oct. 1984.

[24] I. V. Lindell, Methods for Electromagnetic Field Analysis. Piscataway, NJ: IEEE Press, 1995.

[25] J. E. Hipp, "Soil electromagnetic parameters as functions of frequency, soil density, and soil moisture," Proc. IEEE, vol. 62, pp. 98-103, Jan. 1974.

[26] J. A. Kong, Electromagnetic Wave Theory. New York: Wiley, 1990.

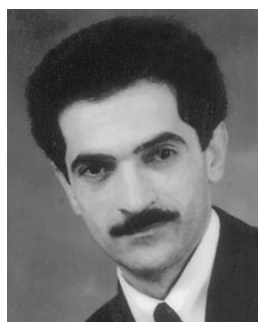

Kamal Sarabandi (S'87-M'90-SM'92-F'00) received the B.S. degree in electrical engineering. from Sharif University of Technology, Tehran, Iran, in 1980. He received the M.S.E. degree in electrical engineering, the M.S. degree in mathematics, and the $\mathrm{Ph} . \mathrm{D}$. degree in electrical engineering from The University of Michigan, Ann Arbor, in 1986, 1989. and 1989 , respectively.

$\mathrm{He}$ is currently the director of the Radiation Laboratory and Professor in the Department of Electrical Engineering and Computer Science, University of Michigan. He has served as the Principal Investigator on many projects sponsored by NASA, JPL, ARO, ONR, ARL, NSF, DARPA, and numerous industries. He has published many book chapters and more than 90 papers in refereed journals on electromagnetic scattering, random media modeling, wave propagation, antennas, microwave measurement techniques, radar calibration, inverse scattering problems, and microwave sensors. $\mathrm{He}$ has also had more than 170 papers and invited presentations in national and international conferences and symposia on similar subjects. His research interests include electromagnetic wave propagation, antennas, and microwave and millimeter-wave radar remote sensing.

Dr. Sarabandi is a member of the IEEE Geoscience and Remote Sensing Society (GRSS) ADCOM, Chairman of the Awards Committee of the IEEE GRSS, and a member of IEEE Technical Activities Board Awards Committee. He is the Associate Editor of the IEEE TRANSACTIONS ON ANTENNAS AND PROPAGATION (TAP) and the IEEE SENSORS JOURNAL. He is also a member of Commission $\mathrm{F}$ of URSI and of The Electromagnetic Academy. He is listed in American Men \& Women of Science and Who's Who in America. He received the Henry Russel Award from the Regent of The University of Michigan. In 1999, he received a GAAC Distinguished Lecturer Award from the German Federal Ministry for Education, Science, and Technology. He also received a 1996 Teaching Excellence Award from the Electrical Engineering and Computer Science Department of The University of Michigan.

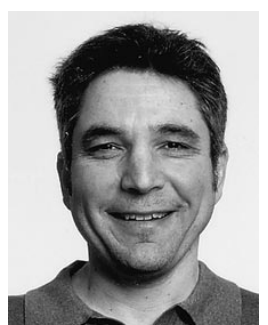

Mark D. Casciato (S'86-M'01) was born in New Castle, PA, in June 1956. He received the B.S.E.E. and M.S.E.E. degrees from Florida Atlantic University, Boca Raton, and the Ph.D. degree from the University of Michigan, Ann Arbor, in 1988, 1995, and 2001, respectively.

From 1989 to 1992, he worked as an RF/Microwave Design Engineer at Allied Signal Aerospace in Ft. Lauderdale, FL. In 1995, he was an Intern at MIT-Lincoln Laboratory/Group 34, working in the area of electromagnetic scattering prediction. Currently, he is an Assistant Research Scientist, with the University of Michigan Radiation Laboratory. His current research interests include electromagnetic scattering and diffraction techniques as applied to radio wave propagation and radar.

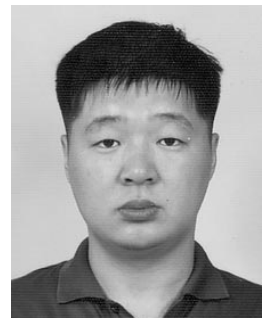

large object.
II-Suek Koh (A'01) was born in Korea. He received the B.S. and M.S. degrees in electronics engineering from Yonsei University, Seoul, Korea, in 1992 and 1994, respectively. He is currently working toward the $\mathrm{Ph} . \mathrm{D}$ degree at the University of Michigan, Ann Arbor.

In 1994, he joined LG Electronics Ltd. Seoul, Korea, as an Assistant Research Engineer. His research interests include theoretical modeling of wireless communication channel, multibody problem, and numerical technique for electrically 